\title{
The Repressor C Protein, Pf4r, Controls Superinfection of Pseudomonas aeruginosa PAO1 by the Pf4 Filamentous Phage and Regulates Host Gene Expression
}

\author{
Muhammad Hafiz Ismail 1,2 ${ }^{10}$, Katharine A. Michie ${ }^{3}$, , Yu Fen Goh ${ }^{1}$, Parisa Noorian ${ }^{4}$, Staffan Kjelleberg ${ }^{1,2}$, \\ Iain G. Duggin ${ }^{4}\left(\mathbb{D}\right.$, Diane McDougald ${ }^{1,4}$ and Scott A. Rice ${ }^{1,2,4, *}$ \\ 1 Singapore Centre for Environmental Life Sciences Engineering, Singapore 637551, Singapore; \\ muhd.hafiz@ntu.edu.sg (M.H.I.); YUFEN001@e.ntu.edu.sg (Y.F.G.); laskjelleberg@ntu.edu.sg (S.K.); \\ Diane.McDougald@uts.edu.au (D.M.) \\ 2 The School of Biological Sciences, Nanyang Technological University, Singapore 637551, Singapore \\ 3 Structural Biology Facility, Mark Wainwright Analytical Centre, The University of New South Wales, \\ Sydney, NSW 2052, Australia; k.michie@unsw.edu.au \\ 4 The iThree Institute, The University of Technology Sydney, Sydney, NSW 2007, Australia; \\ parisa.noorian@uts.edu.au (P.N.); Iain.Duggin@uts.edu.au (I.G.D.) \\ * Correspondence: rscott@ntu.edu.sg
}

check for updates

Citation: Ismail, M.H.; Michie, K.A.; Goh, Y.F.; Noorian, P.; Kjelleberg, S.; Duggin, I.G.; McDougald, D.; Rice, S.A. The Repressor C Protein, Pf4r, Controls Superinfection of Pseudomonas aeruginosa PAO1 by the Pf4 Filamentous Phage and Regulates Host Gene Expression. Viruses 2021, 13, 1614. https://doi.org/10.3390/ v13081614

Academic Editor: Dann Turner

Received: 22 July 2021

Accepted: 13 August 2021

Published: 15 August 2021

Publisher's Note: MDPI stays neutral with regard to jurisdictional claims in published maps and institutional affiliations.

Copyright: (c) 2021 by the authors. Licensee MDPI, Basel, Switzerland. This article is an open access article distributed under the terms and conditions of the Creative Commons Attribution (CC BY) license (https:// creativecommons.org/licenses/by/ $4.0 /)$.
Abstract: It has been shown that the filamentous phage, Pf4, plays an important role in biofilm development, stress tolerance, genetic variant formation and virulence in Pseudomonas aeruginosa PAO1. These behaviours are linked to the appearance of superinfective phage variants. Here, we have investigated the molecular mechanism of superinfection as well as how the Pf4 phage can control host gene expression to modulate host behaviours. Pf4 exists as a prophage in PAO1 and encodes a homologue of the P2 phage repressor C and was recently named Pf4r. Through a combination of molecular techniques, ChIPseq and transcriptomic analyses, we show a critical site in repressor C (Pf4r) where a mutation in the site, 788799A $>\mathrm{G}$ (Ser4Pro), causes Pf4r to lose its function as the immunity factor against reinfection by Pf4. X-ray crystal structure analysis shows that Pf4r forms symmetric homo-dimers homologous to the E.coli bacteriophage P2 RepC protein. A mutation, Pf4r*, associated with the superinfective Pf4r variant, found at the dimer interface, suggests dimer formation may be disrupted, which derepresses phage replication. This is supported by multi-angle light scattering (MALS) analysis, where the Pf4r* protein only forms monomers. The loss of dimerisation also explains the loss of Pf4r's immunity function. Phenotypic assays showed that Pf4r increased LasB activity and was also associated with a slight increase in the percentage of morphotypic variants. ChIPseq and transcriptomic analyses suggest that Pf4r also likely functions as a transcriptional regulator for other host genes. Collectively, these data suggest the mechanism by which filamentous phages play such an important role in P. aeruginosa biofilm development.

Keywords: bacteriophage; gene regulation; biofilm

\section{Introduction}

Bacteriophages are important factors in the diversification of bacterial species. In Corynebacterium diphtheriae and Escherichia coli O157, prophages, bacteriophage integrated into the host chromosome and are replicated as part of the host, have been shown to harbour genes that encode toxins and virulence factors, enhancing the pathogenicity of the host bacterium [1-3]. Endogenous oxidative stress in biofilms is also known to drive diversity in biofilm communities [4]. In Salmonella, a prophage-encoded superoxide dismutase was shown to be the primary oxidative stress defence mechanism for the bacterium [5], thus linking phage to oxidative stress responses in the bacterial host. Bacteriophage can also contribute to the regulation of host genes [6,7]; for example, a loss of virulence of Ralstonia solanacearum and P. aeruginosa was observed when infected by certain bacteriophages. Thus, 
prophages contribute to bacterial fitness and evolution through a combination of horizontal gene exchange, stress response and regulation of gene expression and behaviour through mechanisms such as transcription factors, sRNAs and even bacterial lysis [8,9].

Filamentous phage, Inoviruses, have recently been shown to be widespread in bacteria and archaea, suggesting a long evolutionary relationship with their hosts [10]. P. aeruginosa PAO1 carries two filamentous phage integrated into its genome, Pf4 and Pf6, found in tRNA genes at positions PA0729.1 and PA4673.1 [11,12]. While no phenotype has currently been ascribed to the Pf6 prophage, the Pf4 prophage has been shown to play a crucial role in biofilm development where it influences maturation, cell death, dispersal and variant formation of the biofilm [13-17]. These effects are seen where the Pf4 filamentous phage acquires a superinfective (SI) phenotype [11,13]. Normally, P. aeruginosa carrying a prophage confers resistance to plaque formation (infection) upon exposure to its own phage. However, when the phage acquires particular mutations, that mutant phage can reinfect the host, which we refer to here as superinfective $[14,18]$. Additionally, it was shown that a superinfecting Pf4 mutant was less virulent in a mouse model of lung infection, suggesting that the phage plays a role in mediating virulence in the host $[13,19]$. Unlike phage lambda, the Pf4 prophage continually produces phage particles without resulting in host cell death [20]. Recently, it was observed that SI phages produced by P. aeruginosa have mutations in a gene (located in the intergenic region of PA0716 and PA0717) that has homology to an immunity protein of the $\mathrm{P} 2$ and lambda phage called the repressor C protein [14]. Li et al. [21] demonstrated that repressor C, which they named Pf4r, was responsible for immunity to Pf4 infection and also regulated replication of the Pf4 phage by repressing the expression of xisF4. However, the specific mechanism by which the Pf4r controls superinfection remained unknown in the context of the mutated Pf4r* [14].

Additionally, it has been shown that transcription factors encoded by phage can regulate host gene expression. In enterohemorrhagic E. coli (EHEC), deletion of the bacteriophage regulatory genes, anti-terminators $\mathrm{N}$ and $\mathrm{Q}$ and lytic genes $\mathrm{S}$ and $\mathrm{R}$ decreased the expression of the locus of enterocyte effacement (LEE)-encoded type III secretion system. It was subsequently discovered that the bacteriophage-encoded Cro functioned as a transcriptional activator of LEE as well as host virulence genes responsible for fimbria and flagellar expression [22]. Given that deletion of Pf4 resulted in decreased virulence of $P$. aeruginosa PAO1 [13,19], it is possible that Pf4r also plays a role in the expression of host genes.

The mechanism by which Pf4r contributes to the appearance of superinfection was investigated here. We observed that a mutation at Ser4Pro in Pf4r* was responsible for loss of immunity against reinfection but not at Arg80Leu. Furthermore, structural and biochemical analysis of the Pf4r mutant reveals that the superinfective Pf4r protein is deficient in dimerisation, a structural requirement for effective DNA binding. This loss of dimerisation is likely to explain why the mutant Pf4r protein does not bind its native promoter site. Complementation of Pf4r to the Pf4-deficient mutant restored LasB activity to that of the wild-type P. aeruginosa. In addition, Pf4r contributed to an increase in genetic variation of $P$. aeruginosa and potentially contributes to generating diversity in biofilms, especially in the presence of endogenous oxidative stress. The potential role of Pf4r in regulating gene expression of other genes was also explored using ChIPseq and transcriptomic analysis.

\section{Materials and Methods}

\subsection{Bacterial Strains and Culture Conditions}

All bacterial strains (Table S1) were maintained on Lysogeny Broth (LB) medium (BD Difco, NJ, USA), either in broth or on plates supplemented with $1.5 \%(w / v)$ agar when necessary. Bacteria were cultured in either LB broth or M9 minimal medium ( $48 \mathrm{mM}$ $\mathrm{Na}_{2} \mathrm{HPO}_{4}, 22 \mathrm{mM} \mathrm{KH}_{2} \mathrm{PO}_{4}, 9 \mathrm{mM} \mathrm{NaCl}, 19 \mathrm{mM} \mathrm{NH}_{4} \mathrm{Cl}, 2 \mathrm{mM} \mathrm{MgSO}_{4}$ and $0.1 \mathrm{mM} \mathrm{CaCl}_{2}$, supplemented with $0.2 \%(w / v)$ casamino acids and $0.04 \%(w / v)$ glucose). For plasmid maintenance in E. coli, the medium was supplemented with either $100 \mu \mathrm{g} / \mathrm{mL}$ carbenicillin 
or gentamicin (Carb100 or Gm100). P. aeruginosa PAO1 strains carrying the pJN105 vector and its derivatives were grown in medium supplemented with $100 \mu \mathrm{g} / \mathrm{mL}$ gentamicin (LB Gm100 or M9 Gm100). The cultures were incubated overnight at $37^{\circ} \mathrm{C}$, at $200 \mathrm{rpm}$.

\subsection{Genomic DNA and Plasmid DNA Extraction}

Genomic DNA was extracted using the QIAamp ${ }^{\circledR}$ DNA Mini Kit (Qiagen, Germany), while plasmid DNA was extracted with the FavorPrep plasmid extraction kit (Favorgen, Taiwan) as per the manufacturers' instructions. The concentration was assessed using both a NanoDrop 2000 Spectrophotometer (Thermo Scientific, MA, USA) and Qubit ${ }^{\circledR} 2.0$ Fluorometer (Life Technologies, CA, USA).

\subsection{Polymerase Chain Reaction}

Polymerase chain reactions (PCR) were performed in a Mastercycler pro (Eppendorf, Germany) using either Platinum ${ }^{\mathrm{TM}}$ Taq DNA polymerase (Invitrogen, MA, USA) or Q5 DNA polymerase (New England Biolabs, MA, USA) as per the manufacturers' recommendation and as previously described [23]. The cycling conditions were: (1) initial denaturation at $95^{\circ} \mathrm{C}$ for $3 \mathrm{~min}$; (2) 25-30 cycles of $95^{\circ} \mathrm{C}$ for $30 \mathrm{~s}$, annealing at temperatures as specified (Table S2) [24] for $30 \mathrm{~s}$, and extension at $72{ }^{\circ} \mathrm{C}$ for the time as specified (Table S2) [24] and (3) a final extension at $72{ }^{\circ} \mathrm{C}$ for 5 (Platinum ${ }^{\mathrm{TM}}$ Taq DNA polymerase) or 2 min (Q5 DNA polymerase).

\subsection{Gel Electrophoresis}

Gel electrophoresis analyses were performed using either 1 or $2 \%(w / v)$ agarose dissolved in $1 \times$ TAE buffer (39.95 mM Tris-base, 11.42\% (v/v) glacial acetic acid, $127.29 \mu \mathrm{M}$ EDTA) [23]. One or two microliters of $6 \times$ DNA loading dye (Fermentas Life Sciences, MA, USA) was mixed with $10 \mu \mathrm{L}$ of DNA or $1 \mu \mathrm{L}$ of GeneRuler ${ }^{\mathrm{TM}} 1 \mathrm{Kbp}$ or 100 bp DNA ladder (Fermentas Life Sciences, MA, USA) for loading into the gels and subsequent electrophoresis and image capture (Molecular Imager Gel Doc System, Bio-Rad Laboratories, CA, USA).

\subsection{Site-Directed Mutagenesis (SDM) of Pf4r}

The wild-type (WT) pf4r sequence was amplified with pf4r_ORF_FrontUp_F and pf4r_P_ORF_R primers (Table S2) [24] using Platinum Taq DNA polymerase (Invitrogen, USA) and inserted into the pCR4 plasmid of the TOPO TA Cloning Kit (Invitrogen, USA) before it was transformed into TOP10 cells as per the manufacturer's instructions. Sitedirected mutagenesis (SDM) of the four $p f 4 r$ nucleotide mutations on the resultant plasmid, pCR4_Pf4r, was carried out sequentially (Figure S1a) using the Q5 Site-Directed Mutagenesis Kit (New England Biolabs, MA, USA) according to the manufacturer's instructions and as previously described [23]. All plasmids were transformed into NEB5 $\alpha$ cells through heat shock and grown overnight with constant shaking before being extracted. A plasmid with only the SNP 788826G > T was generated using the primers pf4r_SDM03_G-T_F and pf4r_SDM03_G-T_R to modify this region from pCR4_Pf4r to generate the plasmid pCR4_motifSNP.

\subsection{Electrophoretic Mobility Shift Assay (EMSA) of Pf4r}

The $p f 4 r$ and mutated $p f 4 r$ sequences were sent as targets to the Protein Production Platform (PPP) (Biopolis, Singapore) for expression and purification of C-terminal hexahistidine tagged (CT6His) proteins to be used for electrophoretic mobility shift assay (EMSA). Prior to EMSA, the proteins were diluted in $1 \mathrm{X}$ TE buffer $(10 \mathrm{mM}$ Tris- $\mathrm{HCl} \mathrm{pH}$ 8.0, $1 \mathrm{mM}$ EDTA). All primers used for amplification of EMSA targets were $5^{\prime}$ biotinylated (IDT, Singapore) (Table S2) [24]. The $p f 4 r$ target was amplified with pf4r_EMSA_F and pf4r_EMSA_R while the non-target was amplified with gapA_EMSA_F and gapA_EMSA_R using PAO1 genomic DNA as the template. The $\mathrm{P}_{p f 4 r \text {-doubleSNP }}$ probe, which contained the two SNPs in 
the promoter region, and the $\mathrm{P}_{p f 4 r-\mathrm{G}>\mathrm{T}}$ and $\mathrm{P}_{p f 4 r-\mathrm{A}>\mathrm{G}}$ probes, which contained an SNP in positions 1 and 2, respectively (Table S2).

Protein-DNA complexes were separated from free DNA on $6 \%$ polyacrylamide DNA retardation gels (Invitrogen, MA, USA). All binding and chemiluminescence reactions were carried out with the Pierce LightShift Chemiluminescent EMSA kit (Thermo Scientific, MA, USA) according to manufacturer's instruction. Binding reactions were performed with protein concentrations in lanes 1 to 10 at either 0 or $90 \mathrm{nM}$ and $10 \mathrm{fmol}$ of target probe for $30 \mathrm{~min}$ at $30{ }^{\circ} \mathrm{C}$ in Pf4r binding buffer (10 mM Tris, $1 \mathrm{mM} \mathrm{DTT,} \mathrm{2.5 \%} \mathrm{Glycerol,} 5 \mu \mathrm{g} / \mu \mathrm{L}$ BSA, $0.05 \% \mathrm{NP}-40,80 \mathrm{mM} \mathrm{KCl}, 5 \mathrm{mM} \mathrm{MgCl}{ }_{2}$ and $2 \mathrm{mM}$ EDTA). After incubation, $5 \mu \mathrm{L}$ of 5X Novex Hi-Density TBE Sample Buffer (Thermo Scientific, MA, USA) was mixed with the binding reaction. The protein-DNA complex was then electrophoresed for $120 \mathrm{~min}$ at $110 \mathrm{~V}$ in $0.5 \mathrm{X}$ TBE ( $40 \mathrm{mM}$ Tris-Cl pH 8.3, $45 \mathrm{mM}$ boric acid and $1 \mathrm{mM}$ EDTA) buffer through a $6 \%$ polyacrylamide gel at $4{ }^{\circ} \mathrm{C}$.

\subsection{SEC-MALS Analysis of Purified Pf4r and Pf6r Proteins}

SEC-MALS was performed according to previously established methods [25]. Briefly, Pf4r, Pf4r* and Pf6r samples (prepared in $20 \mathrm{mM}$ HEPES pH 7.5, $300 \mathrm{mM} \mathrm{NaCl}$, glycerol $10 \%(v / v), 2 \mathrm{mM}$ TCEP) were gel-filtrated on a Superdex $755 / 150$ gel filtration column (GE Healthcare, IL, USA) equilibrated with PBS. The chromatography system was connected in-line to a miniDAWN light scattering unit (Wyatt Technology, CA, USA) and an Optilab T-rEX differential refractive index detector (Wyatt Technology, CA, USA). Loading volumes were $50 \mu \mathrm{L}$ with protein concentrations of $20 \mathrm{mg} / \mathrm{mL}$. Data were analysed in Astra 6 (Wyatt Technology, CA, USA).

\subsection{Crystal Structure of the Pf4r* Protein}

The crystallography of Pf4r* was performed using previously described methods [26]. For crystallization, an initial screening of Pf4r $(29 \mathrm{mg} / \mathrm{mL}), \mathrm{Pf} \mathrm{r}^{*}(50 \mathrm{mg} / \mathrm{mL})$ and Pf6r $(17 \mathrm{mg} / \mathrm{mL})$ proteins were performed in buffer containing $20 \mathrm{mM}$ HEPES $\mathrm{pH}$ 7.5, $1 \mathrm{mM}$ EDTA, $2 \mathrm{mM}$ TCEP, $300 \mathrm{mM} \mathrm{NaCl}, 10 \%$ glycerol and $100 \mathrm{~nL}$ of precipitant solution from a number of commercial crystallisation screens in sitting drop trays. Crystals for both Pf4r $^{*}$ and Pf6r were isolated and optimised prior to data collection at the Australian Synchrotron on MX1. Crystals were frozen in precipitant solution with a 20-25\% PEG200 cryoprotectant. Pf4r* crystals were soaked with a number of heavy atom derivatives and SAD data at a wavelength of $1.0055 \AA$ was successfully collected on a Mersayl (mercury) soak. Crystal trays contained $50 \mathrm{mg} / \mathrm{mL}$ Pf4r*, 37\% PEG3350, $50 \mathrm{mM}$ Tris pH 7, $50 \mathrm{mM} \mathrm{KCl}$ and 5\% glycerol. Phasing was performed in AUTOSOL [27] within the Phenix software package, and data refinement was performed in Phenix [28]. Building was carried out in Coot [29]. Pf4r* was built and refined at $2.7 \AA$ using the SAD data. Once refinement was complete, this model was used for Molecular Replacement (using Phaser [30]) to solve the structures of Pf4r* at $1.99 \mathrm{~A}$ and Pf6r at $1.73 \AA$ on crystals in the following conditions-50 mg/mL Pf4r*, PEGMME 550 25\%, $50 \mathrm{mM}$ HEPES pH 7.0, $10 \mathrm{mM} \mathrm{MgCl}_{2}$; and $17.7 \mathrm{mg} / \mathrm{mL}$ Pf6r, polyethene glycol monomethyl ether $500020 \%$, ammonium nitrate $200 \mathrm{mM}, 25 \%$ PEG 200. Analysis of the final models in PDBePISA [31,32] indicates that both proteins form various dimers within the crystal. See Table S3 for crystallographic data collection and refinement statistics.

\subsection{Tubing Biofilm System for Phenotypic Assays and mRNA Analysis}

Biofilms were allowed to develop for $2 \mathrm{~d}$ at room temperature in $15 \mathrm{~cm}$ long silicon tubing size $3.18 \mathrm{~mm}$ (Silastic ${ }^{\circledR}$ laboratory tubing, Dow Corning, MI, USA) in a continuous flow reactor setup under sterile conditions. The biofilm system was fed with M9 minimal salts medium. During inoculation of the bacterial culture, the pump was switched off, and the upstream tubing was clamped to prevent backflow. Using a $3 \mathrm{~mL}$ syringe with a $26 \mathrm{G} \times 1 \frac{1}{2}$ " needle, $2 \mathrm{~mL}$ of $1 \times 10^{8}$ cells $/ \mathrm{mL}$ bacterial culture was injected into the lumen of the tubing. The bacterial cells were allowed to attach to the tubing surface for $1 \mathrm{~h}$ at 
room temperature under static conditions. The tubing was then unclamped, and flow was restored at a flow rate of $9 \mathrm{~mL} / \mathrm{h}$.

\subsection{Pyoverdine and Pyocyanin Production}

For planktonic cultures, bacteria were inoculated in M9 minimal medium and incubated overnight at $37^{\circ} \mathrm{C}$, at $200 \mathrm{rpm}$. For biofilms, effluent was collected daily and filtered through a $0.2 \mu \mathrm{m}$ filter. From the cell-free supernatant, pyoverdine was semi-quantified as previously described [33] by measuring the emission fluorescence (Ex: $400 \mathrm{~nm}, \mathrm{Em}$ : $460 \mathrm{~nm})$ while pyocyanin was semi-quantified by absorbance $\left(\mathrm{OD}_{695}\right)$.

\subsection{Production of Secreted Proteases}

To determine levels of proteolytic activity, cell-free supernatant was added to elastincongo red to measure LasB activity [34]. Briefly, cell-free supernatant was added to elastincongo red dissolved in reaction buffer $1\left(0.05 \mathrm{M}\right.$ Tris- $\left.\mathrm{HCl}, 0.5 \mathrm{mM} \mathrm{CaCl}_{2}, \mathrm{pH7.5}\right)$. The elastin-congo red mixture was incubated at $37^{\circ} \mathrm{C}$ for $2 \mathrm{~h}$ with shaking, and reaction stopped by adding EDTA to a final concentration of $10 \mathrm{mM}$. The mixture was pelleted, and supernatant was transferred to microtiter plate to measure absorbance at $\mathrm{OD}_{495}$.

\subsection{Estimation of Replication Fidelity and $\mathrm{H}_{2} \mathrm{O}_{2}$-Induced Mutant Frequencies}

Overnight cultures were diluted to $\mathrm{OD}_{600} 0.1$ and allowed to grow to mid-log $\left(\mathrm{OD}_{600} 0.5\right)$. The cultures were washed with $0.9 \% \mathrm{NaCl}$ twice before being treated with $25 \mathrm{mM}$ and $50 \mathrm{mM}$ of $\mathrm{H}_{2} \mathrm{O}_{2}$ for $30 \mathrm{~min}$ at $37{ }^{\circ} \mathrm{C}$ [35]. The treated cells were washed twice with $0.9 \% \mathrm{NaCl}$, serial diluted, spotted onto LB plates and incubated overnight at $37^{\circ} \mathrm{C}$ to determine cell viability. To determine the $\mathrm{H}_{2} \mathrm{O}_{2}$-induced mutant frequency, $500 \mu \mathrm{L}$ of treated and washed cells were inoculated into $4.5 \mathrm{~mL}$ of $\mathrm{LB}$ media and cultured overnight at $37^{\circ} \mathrm{C}$. Appropriate dilutions of the overnight cultures were then plated on LB plates and incubated overnight at $37^{\circ} \mathrm{C}$ before enumerating the number of standard and variant colonies.

\subsection{Construction and Expression of C-Terminal Hexahistidine Tagged Pf4r}

Construction of plasmids and transformations were performed using standard methods as previously described [23]. The $p f 4 r$ was amplified with pf4r_F_PstI and pf4r_R_CT6His _XbaI, and pf4r_SDM01_F_PstI and pf4r_R_CT6His_XbaI (Table S2) [24] primers, respectively, and ligated into the PstI-XbaI site of pBAD_yhjH, which has a pJN105 plasmid backbone, to generate the pPf4r and pPf4r* plasmid, respectively. The yhjH gene was excised with the digestion of $\mathrm{pBAD}$-yhjh using PstI and $\mathrm{XbaI}$ restriction endonucleases. The pPf4r and pPf4r* plasmids were transformed into E. coli TOP10 cells by heat shock, grown overnight and extracted. To generate $\mathrm{pPf}_{4} \mathrm{r}_{\mathrm{A}>\mathrm{G}}$ and $\mathrm{pPf} 4 \mathrm{r}_{\mathrm{C}>\mathrm{A}}$, primers Pf4r_SDM1_AtoG_F and Pf4r_SDM1_AtoG_R and Pf4r_SDM2_CtoA_F and Pf4r_SDM2_CtoA_R were used with the Q5 Site-Directed Mutagenesis Kit (New England Biolabs, USA), respectively. The pJN105 plasmid backbone had a gentamicin resistance cassette. Therefore, to prevent selection of false-positive clones, the gentamicin resistance cassette in PAO1 $\Delta \mathrm{Pf} 4:: \mathrm{Gm}^{\mathrm{r}}$ was excised. Briefly, the pFLP2 plasmid was electroporated into PAO1 $\triangle \mathrm{Pf} 4:: \mathrm{Gm}^{\mathrm{r}}$ and spread on LB Carb100 plates after recovery. Transformants were then plated on LB agar with $10 \%$ sucrose $(w / v)$ to counter select for the plasmid pFLP2. To ensure that the gentamicin resistance cassette was excised, transformants were then spread onto LB Gm100 plates to test for resistance. Transformants that could not grow on LB Gm100 plates were labelled as $\mathrm{PAO} 1 \triangle \mathrm{Pf} 4$ for the insertion of extracted pPf4r, $\mathrm{pPf} 4 \mathrm{r}_{\mathrm{A}>\mathrm{G}}, \mathrm{pPf}_{\mathrm{P}} \mathrm{r}_{\mathrm{C}>\mathrm{A}}$ and $\mathrm{pPf} 4 \mathrm{r}^{*}$ plasmids. The empty pJN105 plasmid was transformed into P. aeruginosa PAO1 as a control to produce PAO1 $\triangle$ Pf4 pJN105.

\subsection{In Vitro Immunity Assay of Pf4r Protein}

Filtered phage effluent was obtained from overnight cultures of PAO1 by passing through a $0.2 \mu \mathrm{m}$ Acrodisc syringe filter (Pall Life Sciences, NY, USA). Each filtered phage 
effluent was serially diluted for determination of Plaque Forming Units (PFU) using a modified top layer agar (TLA) method previously described by Webb et al. [36]. TLA plates were made by seeding the LB agar top layer with $5 \mathrm{~mL}$ LB soft agar $(0.75 \% w / v)$ containing $750 \mu \mathrm{L}$ of PAO1, PAO1 $\Delta \mathrm{Pf} 4$ or PAO1 $\Delta$ Pf 4 complemented with $\mathrm{pPf} 4 \mathrm{r}, \mathrm{pPf} 4 \mathrm{r}_{\mathrm{A}>\mathrm{G}}, \mathrm{pPf} 4 \mathrm{r}_{\mathrm{C}>\mathrm{A}}$ and $\mathrm{pPf} 4 \mathrm{r}^{*} \mathrm{M} 9$ medium overnight culture. Ten microliters of each effluent dilution were spotted in triplicate onto TLA plates with PAO1, PAO1 $\triangle \mathrm{Pf} 4$ and PAO1 $\triangle \mathrm{Pf} 4$ complemented with pPf4r, $\mathrm{pPf}^{\circ} \mathrm{r}_{\mathrm{A}>\mathrm{G}}, \mathrm{pPf} 4 \mathrm{r}_{\mathrm{C}>\mathrm{A}}$ and $\mathrm{pPf} 4 \mathrm{r}^{*}$ lawns. The plates were dried and incubated overnight at $37^{\circ} \mathrm{C}$. The PFU per $\mathrm{mL}$ for each sample was obtained from dilutions yielding plaque counts between 10 and 100 plaques per spot.

\subsection{Quantification of pf4r Expression by $q R T-P C R$}

The levels of gene expression of $p f 4 r$ in PAO1, PAO1 $\triangle \mathrm{PF} 4, \mathrm{PAO} 1 \Delta \mathrm{Pf} 4 \mathrm{pJN} 105$ and PAO1 $\triangle \mathrm{Pf} 4$ pPf4r was determined using qRT-PCR and the primers pf4r_qPCR_F and pf4r_qPCR_R (Table S2) $[23,24]$. A standard curve was generated from the reactions carried out with serial dilutions of $p f 4 r$ amplicon amplified using the same primers as the qPCR. Each qRT-PCR reaction mixture contained: $10 \mu \mathrm{L}$ of 2 X Fast SYBR ${ }^{\circledR}$ Green Master Mix (Applied Biosystems, MA, USA) added to $0.2 \mu \mathrm{L}$ of forward and reverse primers, respectively, and $1 \mu \mathrm{L}$ of template DNA before being topped up to a final volume of $20 \mu \mathrm{L}$ with deionised water. All qRT-PCR runs were performed using the StepOnePlus ${ }^{\mathrm{TM}}$ Systems (Life Technologies, CA, USA). The cycling conditions were: pre-cycling at $95^{\circ} \mathrm{C}$ for $2 \mathrm{~min}$, followed by 40 cycles of $95^{\circ} \mathrm{C}$ for $10 \mathrm{~s}$ and annealing and extension at $60^{\circ} \mathrm{C}$ for $30 \mathrm{~s}$. Results were analysed using the StepOnePlus ${ }^{\mathrm{TM}}$ Software version 2.3 (Life Technologies, CA, USA), using the standard curve to quantify the amount of $p f 4 r$ transcript in each sample.

\subsection{Chromatin Immunoprecipitation Sequencing (ChIPseq) Sample Preparation}

The ChIPseq protocol used here was modified from Bonocora and Wade [37]. Briefly, PAO1 pJN105, PAO1 pPf4r and PAO1 pPf4r* was inoculated in LB and incubated overnight at $37^{\circ} \mathrm{C}$, at $200 \mathrm{rpm}$ in a rotary incubator. The following day, the overnight culture was diluted to a final $\mathrm{OD}_{600}$ value of 0.1 and a volume of $42 \mathrm{~mL}$ in a sterile flask. The diluted culture was incubated in a $37^{\circ} \mathrm{C}$ rotary incubator to an $\mathrm{OD}_{600}$ of 0.3 before the addition of L-arabinose to a final concentration of $0.25 \%(w / v)$ with further incubation for $1 \mathrm{~h}$. Molecular grade formaldehyde (Pierce Protein Biology, MA, USA) was then added to a final concentration of $1 \%$ and incubated at room temperature to crosslink any protein-DNA complexes. After incubation, glycine was added to final concentration of $0.5 \mathrm{M}$ to neutralise any free formaldehyde in the medium. The culture was then pelleted and washed 3 times with $1 \times$ TBS (50 mM Tris- $\mathrm{Cl}$, pH 7.5, $150 \mathrm{mM} \mathrm{NaCl}$ ) before finally being pelleted in a $1.5 \mathrm{~mL}$ tube. The pellet was resuspended in $1 \mathrm{~mL}$ FA lysis buffer ( $50 \mathrm{mM}$ HEPES-KOH, $0.1 \%$ sodium deoxycholate, $0.1 \%$ SDS, $1 \mathrm{mM}$ EDTA, $1 \%$ Triton-X100 and $150 \mathrm{mM} \mathrm{NaCl}$ ) containing $4 \mathrm{mg} / \mathrm{mL}$ lysozyme and incubated for $30 \mathrm{~min}$ at $37^{\circ} \mathrm{C}$ followed by $5 \mathrm{~min}$ on ice.

To completely lyse the cell pellet and shear the genomic DNA, the samples were sonicated using a VCX 750 probe sonicator (Sonics \& Materials Inc., CT, USA) with the program of a $30 \mathrm{~s}$ on-off cycle for $30 \mathrm{~min}$ at $40 \%$ amplitude. After sonication, the lysate was transferred into a $1.5 \mathrm{~mL}$ tube on ice before being centrifuged for $5 \mathrm{~min}$ at $21,130 \times \mathrm{g}$ at $4{ }^{\circ} \mathrm{C}$ to pellet cell debris. The supernatant was transferred to a $15 \mathrm{~mL}$ tube containing $2.5 \mathrm{~mL}$ prechilled FA lysis buffer and mixed by inversion.

The supernatant, containing the DNA-protein complexes, was then divided into three, $800 \mu \mathrm{L}$ aliquots to which $20 \mu \mathrm{L}$ of ChIP-Grade Protein A/G Magnetic beads (Pierce Protein Biology, MA, USA) and $20 \mu \mathrm{L}$ of anti-His-tag Rabbit mAb (Cell Signalling Technology, MA, USA) were added. This mixture was incubated overnight on a Labquake ${ }^{\mathrm{TM}}$ rotisserie (Thermo Fisher Scientific, MA, USA) at $4{ }^{\circ} \mathrm{C}$. Protein A/G Magnetic beads and associated protein-DNA complexes were recovered by placing tubes on a magnetic rack for $2 \mathrm{~min}$ or until the supernatant had cleared. The supernatant was discarded and $700 \mu \mathrm{L}$ FA lysis buffer was added and incubated for $3 \mathrm{~min}$ at room temperature on the Labquake rotisserie. 
This washing procedure was repeated with 2 rounds of $750 \mu \mathrm{L}$ FA lysis buffer followed by 2 rounds of $750 \mu \mathrm{L} 10 \mathrm{mM}$ Tris-HCl, $\mathrm{pH} 7.5$.

The beads were recovered using the magnetic rack, and DNA-protein complexes were eluted by addition of $100 \mu \mathrm{L}$ ChIP elution buffer (50 mM Tris- $\mathrm{HCl}, \mathrm{pH} 7.5,1 \%$ SDS and $10 \mathrm{mM}$ EDTA) with incubation at $65^{\circ} \mathrm{C}$ for $10 \mathrm{~min}$ with shaking at $500 \mathrm{rpm}$. The beads were removed by magnetic separation, and the supernatant was boiled to separate the DNA-protein complexes. DNA was recovered and purified using the Zymo ChIP DNA Clean \& Concentrator (Zymo Research, CA, USA) as per the manufacturer's instructions. The DNA was quantified with a Qubit Fluorometer (Thermo Fisher Scientific, MA, USA), and the quality was assessed with a High Sensitivity D1000 ScreenTape (Agilent, CA, USA) before the DNA was sequenced as 75 bp paired-end reads on an Illumina MiSeq platform.

\subsection{ChIPseq Analysis}

The quality of the ChIPseq reads was assessed using FastQC version 0.11 .5 before it was adapter and quality trimmed using BBMap version 36.38 [38]. The data analysis was continued using the trimmed reads in $R$ through the systemPipeR (version 1.12.0) package for pipeline control. The reads were aligned to the P. aeruginosa PAO1 genome using Bowtie2 version 2.2.6, and the output SAM file was converted to an indexed and sorted BAM file. In order to perform the peak calling, the MACS2 (version 2.1.1.20160309) [39] 'callpeak' function was used with the reads from P. aeruginosa pPf4r as the treatment file, and the P. aeruginosa pJN105 reads as the control. The identified peaks were annotated using the ChIPseeker package (version 1.14.2), and the sequences of the peaks were extracted using the GenomicRanges package (version 1.30.3) to facilitate motif discovery. The motif discovery was performed using the online tool MEME-ChIP [40], and the options used were set for an expected motif site distribution of $\geq 1$ occurrence. Finally, the top-scoring motif was used to screen the P. aeruginosa genome to identify genes that may putatively be regulated by Pf4r using the online tool Virtual Footprint (version 3.0) [41].

\subsection{Transcriptomic Analysis of Pf4r Complemented Biofilms}

P. aeruginosa wild type, PAO1 $\Delta \mathrm{Pf} 4$ and PAO1 $\Delta \mathrm{Pf} 4 \mathrm{pPf} 4 \mathrm{r}$ biofilms were formed for $2 \mathrm{~d}$ before the biomass was treated with RNAprotect Bacteria Reagent (Qiagen, Germany) and subsequently extracted using the RNeasy ${ }^{\circledR}$ Mini Kit (Qiagen, Germany) inclusive of in-column DNA digestion. To ensure improved removal of contaminating DNA, the samples were further treated with TURBO ${ }^{\mathrm{TM}}$ DNase (Ambion ${ }^{\circledR}, \mathrm{TX}, \mathrm{USA}$ ), and DNase removal and RNA clean up were performed using RNA Clean \& Concentrator ${ }^{\mathrm{TM}}$ (Zymo Research, CA, USA) as per the manufacturers' instruction. The RNA was converted into cDNA and sequenced as 100 bp paired-end reads using the Illumina HiSeq platform.

The total raw RNA reads were adapter and quality trimmed using BBMap version 36.38 [38] before the rRNA reads were depleted using SortMeRNA version 2.1 [42] to obtain mRNA reads. The mRNA reads were mapped using Bowtie 2 against the P. aeruginosa PAO1 genome (NC_002516) modified to include the Pf6 prophage genome. HTSeq [43] was used to count the number of reads in each feature with the modified genome as a reference. Differentially expressed genes that were statistically significant were identified using the DESeq2 package [44] in R, and the datasets were tested using PERMANOVA to test for significant differences between the different conditions (strain and day). The log2 fold change values extracted at the end were based on the false discovery rate cutoff of 0.1 .

\section{Results}

The effluent of biofilms formed by wild-type P. aeruginosa PAO1 and its isogenic, Pf4 deficient mutant (PAO1 $\Delta \mathrm{Pf} 4)$ were plated onto lawns of the wild type or the $\Delta \mathrm{Pf} 4$ mutant. As previously observed, phage were detected on the PAO1 $\Delta \mathrm{Pf} 4$ mutant lawn at all time points for the wild-type biofilm effluent (Figure 1). The plaque morphology observed is consistent with morphologies observed from previous studies of the Pf4 phage [13,36]. On day 4, we observed plaque formation on the wild-type lawn from the wild-type biofilm 
effluent, suggesting conversion to the superinfective (SI) form (Figure 1a). While the effluent from PAO1 $\triangle$ Pf4 initially produced no plaques, on days 6 and 7 , similar numbers of plaques were observed on both the wild-type and PAO1 $\triangle \mathrm{Pf} 4$ lawns (Figure 1c). Given that PAO1 carries the Pf6 phage in addition to the Pf4, we hypothesised that plaque formation from the PAO1 $\Delta \mathrm{Pf} 4$ mutant was due to the conversion of the Pf6 into the SI form. Quantification of both Pf4 and Pf6 using phage specific primers showed the presence of both phage in the wild-type biofilm effluent and only the Pf6 phage in the PAO1 $\Delta$ Pf4 mutant (Figure 1b,d). For both the Pf4 and Pf6, there was an increase in phage numbers at the time the SI phage were observed on the lawns.

Biofilm effluent

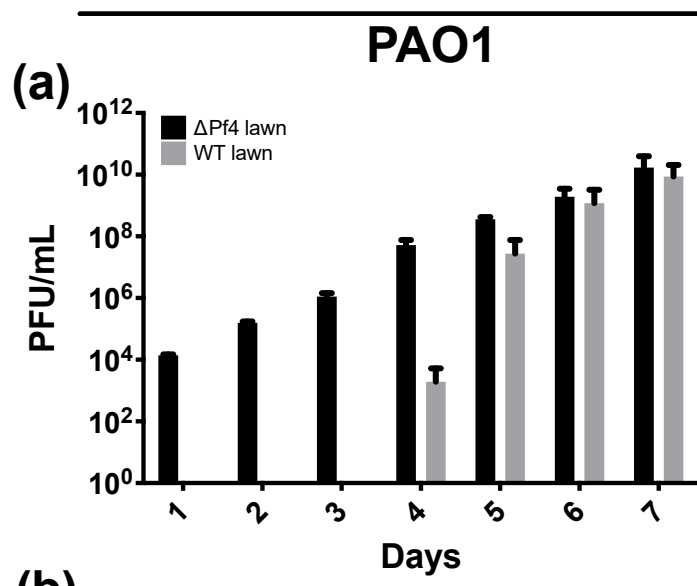

(b)

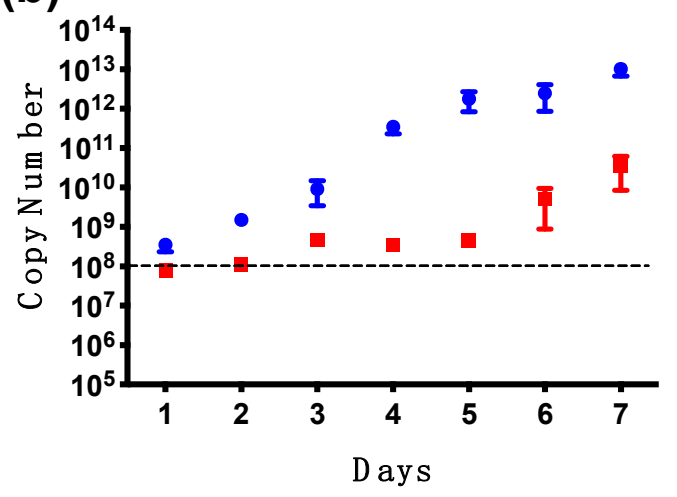

(c)

$\Delta \mathrm{Pf4}$

(d)

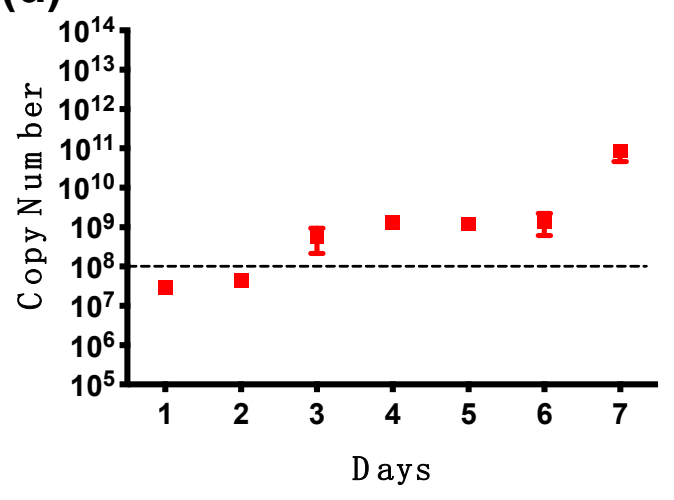

Figure 1. Phage production from biofilms formed by wild-type PAO1 $(\mathbf{a}, \mathbf{b})$ and the PAO1 $\triangle \mathrm{Pf} 4$ mutant (c,d). The bars in black $(\square)$ represent the phage titre of the biofilm on a PAO1 $\triangle$ Pf4 top layer agar, while the bars in grey $(\square)$ represent the biofilm phage titre on a PAO1 top layer agar $(\mathbf{a}, \mathbf{c})$. The points in blue $(\bullet)$ and in red $(\square)$ indicate the amounts of Pf4 or Pf6 DNA, respectively, detected in the biofilm effluent $(\mathbf{c}, \mathbf{d})$. The amounts of phage DNA are represented as the calculated copy numbers and the limit of detection of $1 \times 10^{8}$ (indicated by the dashed line, - ). The biofilms were developed over $7 \mathrm{~d}$. The error bars represent standard deviations. For all figures, there were 3 biological replicates with 3 technical replicates for each, i.e., $N=3$ and $n=3$.

\subsection{The Structure of the Pf4r Protein}

Both the Pf4 and Pf6 phage encode a small protein with homology to the P2 replication repressor, repressor C (RepC). We previously showed that the SI variant of Pf4 has mutations in Pf4r [45]. The mutated Pf4r, with non-synonymous mutations, will be referred to here as Pf4r*. Given that Pf4r shows homology to the P2 repressor C of E. coli, which functions as a DNA-binding protein, we speculated that Pf4r may similarly bind DNA to control the biofilm and lung infection phenotypes previously reported [14,21] and that those mutations might change the structure of Pf4r to alter its ability to bind DNA. We recombinantly over-expressed and purified Pf4r, Pf4r* (containing the mutations Ser4Pro 
and Arg80Leu) and a related repressor from the Pf6 phage (Pf6r), all with a C-terminal hexa-histidine tag for purification. We did not obtain crystals of the native Pf4r of sufficient quality to obtain a high-resolution structure of the protein. However, we were successful in generating high-quality crystals for the Pf4r* mutant from which the structure was directly determined and from which the Pf4r structure was modelled. The Pf4r* structure was solved initially by single anomalous dispersion (SAD) and then by molecular replacement (MR) to a final resolution of $1.97 \AA$ in the space group C 22 21, yielding an asymmetric unit containing two protein chains (A and B). Each protein monomer is comprised of six alpha helices (Figure 2). The first three helices form a helix-turn-helix (HTH) domain. The crystal structure was assessed for biologically relevant dimerisation using the PDBePISA server [31,32], and two independent states of Pf4r* dimerisation were identified, Type A and Type B. Both are symmetric homodimers, each formed by the same 2-fold rotational axis, i.e., the type $\mathrm{A}$ homodimer is formed by two chain $\mathrm{A}$ monomer subunits rotated $180^{\circ}$ to each other, and the Type B homodimer is formed by two chain B monomer subunits rotated around the same axis as Type A (Figure 2c). The complexes have Complex Formation Significance Scores (CSS) of 1.00 and surface areas of $763 \AA^{2}$ and $733 \AA^{2}$, respectively (as determined by PDBePISA), and a structural alignment to each other of RMSD 1.693 (determined using Super within Pymol [46]).

(a)

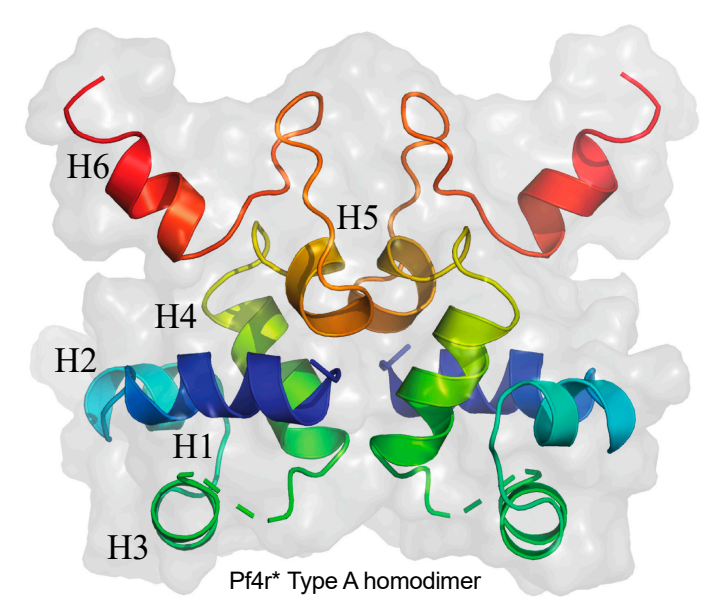

(b)

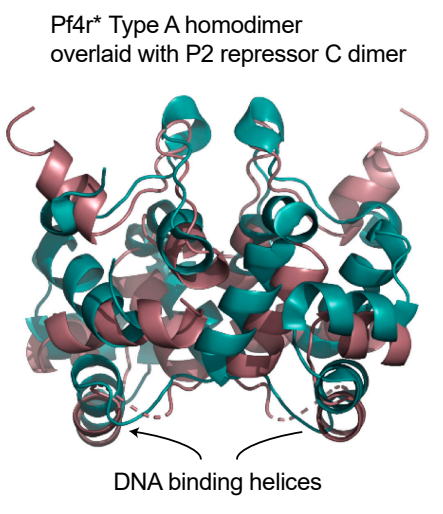

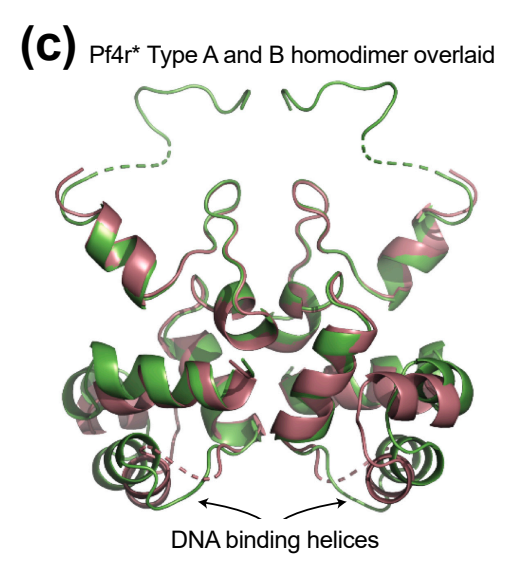

Figure 2. Crystal structure of Pf4r*. (a) The structure is coloured from the N-terminus (blue) to the C-terminus (red) in ribbon representation showing the symmetrical dimer formed by two chain As. The helices are numbered H1-H6, and the surface is rendered in transparent grey. (b) The Pf4r* Type A homodimer structure (brown) overlaid with the distantly related E. coli P2 repressor C (cyan) (PDB 2XCJ), showing high conservation in structure. (c) Overlay of the two types of Pf4r* symmetric homodimer (Type A in brown, Type B in green) observed in the solved crystal. Differences arise within the spacing of the predicted DNA-binding helices. 
Pf4r shares only $27 \%$ amino acid identity with the distantly related phage P2 repressor C protein from E. coli (PDB 2XCJ). Nonetheless, the two symmetric Pf4r homodimer structures each have a homologous structure to the $\mathrm{P} 2$ repressor $\mathrm{C}$ (Figure $2 \mathrm{~b}$ showing the Type A Pf4r* in brown superimposed on the P2 repressor C structure in cyan). The two proteins have very similar folds (RMSD 2.347 calculated using 'Super' within Pymol). The RMSD for an alignment of the Type B Pf4r* complex and the P2 repressor C is 2.625.

The two Helix 3s located at the bottom of the A-type complex of Pf4r* overlay closely with the equivalent Helix 3s from the P2-repressor C protein (Figure 2b). The H3 helices in the $\mathrm{P} 2$ repressor $\mathrm{C}$ are the DNA-binding helices that bind across two turns of the major groove, suggesting that Pf4r may also bind DNA in this fashion. In comparing the two different conformational variants of Pf4r* dimer structures (Type A and Type B) observed in the crystals (Figure 2c), we note two major differences between the two different complexes. In the Type B complex, the recombinant hexa-histidine tag is fully resolved, forming an extended loop at the top of the structure. This is of no biological importance and is not discussed further. The second major difference between the complexes is within the distances between the putative DNA-binding H3 helices (Figure 2b,c). Based on the sequence identity between Pf4r* and Pf4r, it is expected that Pf4r also binds DNA via these two helices, and the variation between the two complexes (Type A and Type B) suggests that there is some plasticity within the loops that orient DNA-binding helices. Similar to the $\mathrm{P} 2$ repressor $\mathrm{C}$, the Pf4r binds to a non-palindromic DNA site, further accentuating the structural similarities between the two distinct proteins. Based on these similarities, we propose that for both Pf4r and the P2 repressor C, the plasticity of the helix placement contributes to the binding of non-identical DNA sites.

Crystals of the related wild-type Pf6r ( $1.73 \AA$ ) ( $51 \%$ identity to Pf4r) had three complexes in the asymmetric unit cell; however, structural alignments of these complexes showed almost no differences between each other (pairwise structural alignments of the three complexes calculated by Super Pymol range between an RMSD of 0.235 and 0.266 ) and the DNA-binding helices have the same spacing as that of Pf4r* Type A and the P2-repressor C protein (Figure S2 and Table S3).

\subsection{Mutations in the Dimerisation Interface Causes Loss of Immunity Function}

The two mutations isolated in Pf4r* were highlighted in the structure (Figure 3a). An Arg to Leu substitution at position 80 (Figure 3a, blue spheres) maps to an exposed surface of the protein on the opposite side of the dimer complex from the predicted DNA-binding motif and also well away from the dimerisation interface. We do not predict this mutation to affect DNA binding or dimerisation, but it is possible it may affect some protein-protein interaction with a currently unknown partner. Changing from Arg to Leu significantly alters the charge state of the surface. We note that in the $\mathrm{P} 2$ repressor $\mathrm{C}$, a 9 amino acid deletion from the C-terminus (which would include the Arg/Leu 80) was fully functional, suggesting this region of the protein is not important for function [47].

The second mutation, a Ser to Pro mutation at residue 4 (78879A $>\mathrm{G})$, was observed to reside directly at the dimer interface (Figure 3a, pink spheres). Aligning the Pf4r* structure with the closely related wild-type Pf6r structure (Figure 3b, bottom left), we can see that the proline mutation has not caused a large structural perturbation, i.e., the two structures (one wild type that dimerises and one being the mutant that does not dimerise in solution) (using SUPERPOSE v1.0) [48] are remarkably similar (RMSD 0.518 for backbone atoms) while being only $49 \%$ identical at the sequence level. Prolines typically break helices and lack hydrogen bonding potential on the $\alpha$ amino group and thus have reduced hydrogenbonding potential. In the Pf $4 r^{*}$ structure (Figure $3 b$ ), if we model a serine in place of the mutated proline to understand the wild-type case, there is a neighbouring aspartate residue across the binding interface on the opposite chain with which the serine residue is able to make two stabilising hydrogen bonds (Figure $3 \mathrm{~b}$, bottom right). The mutation in Pf4r* to proline at position 4 may mean that only weaker hydrophobic interactions can be made, and the homodimerisation may be less stable. While dimerisation was observed in the 
crystals, we believe dimerisation is an artefact of very high protein concentrations required for crystallisation.

(a)

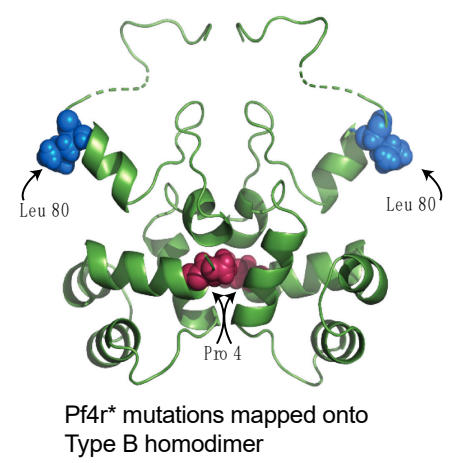

(b)

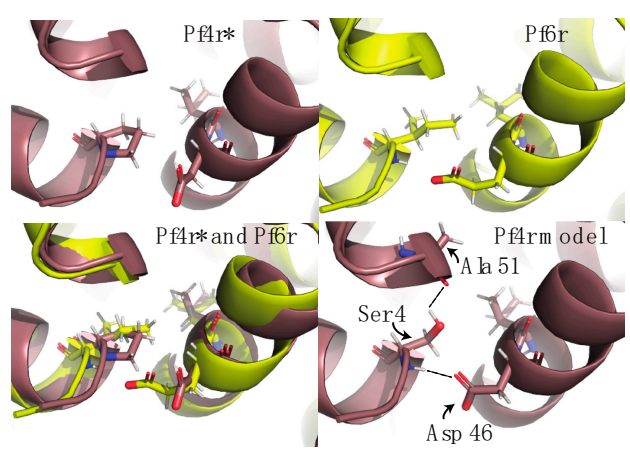

(c)

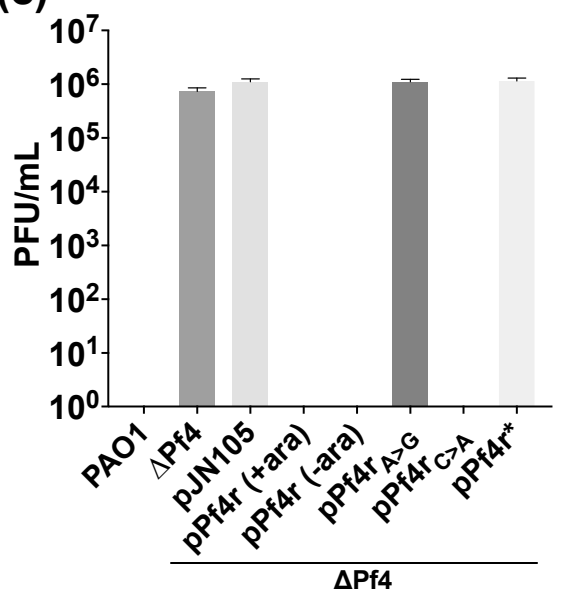

Figure 3. Mutation causing Ser4Pro causes loss of function as immunity factor. (a) Space-filling spheres for the two mutations in Pf4r* that stop DNA binding. Leu 80 (blue) is located on the top outside face of the complex and is unlikely to be involved in DNA binding or dimerisation. Proline 4 (pink) is located at the dimerisation interface and may perturb dimerisation. (b) Detailed view of the proline residue and the Asp 46 from the opposing dimer chain in close proximity in the Pf4r* crystal complex (top left) and the same region in Pf6r (top right). These sections are overlaid (bottom left). Bottom right shows that the wild-type Pf4r Ser4 residue modelled into the Pro4 position of Pf4r* may be able to make hydrogen bonds with the Asp46 on the opposing chain and form a stabilising interaction. Distances show hydrogen to oxygen distances in Angstrom for the two potential hydrogen bonds as determined in Pymol using the mutagenesis tool and measurement wizard. (c) Phage effluent from P. aeruginosa PAO1 was spotted onto different bacterial lawns, and the PFUs were enumerated. $N=3$ and $n=3$.

It was already determined that Pf4r was responsible for conferring immunity to Pf4 infection [21]. However, the same was not determined for Pf6r. Despite our best efforts using multiple approaches, including that which was used to generate the PAO1 $\Delta \mathrm{Pf} 4$ mutant, we could not delete the Pf6 prophage (data not shown). Hence, the rest of the study is focused on the Pf4 phage and Pf4r. To determine the role of the specific mutations in Pf4r* in the loss of immunity, plaque assays were conducted for wild-type PAO1, the mutant lacking the Pf4 phage (PAO1 $\Delta \mathrm{Pf} 4)$, as well as the phage deletion mutant that has been transformed with a plasmid carrying the wild-type $p f 4 r$ or the mutations described above (PAO1 $\triangle$ Pf4 pPf4r, pPf4r $\mathrm{r}_{\mathrm{A}>\mathrm{G}}, \mathrm{pPf} \mathrm{r}_{\mathrm{C}>\mathrm{A}}$ or $\left.\mathrm{pPf} 4 \mathrm{r}^{*}\right)$. The ectopic expression of Pf4r and its mutants were induced using $0.25 \%(w / v)$ L-arabinose. As expected, supernatant from the wild-type $P$. aeruginosa-generated plaques $\left(7.33 \times 10^{5} \mathrm{PFU} / \mathrm{mL}\right)$ on the Pf4 deletion mutant and for the strain carrying the empty vector (PAO1 $\Delta$ Pf4 pJN105) $\left(1.1 \times 10^{6} \mathrm{PFU} / \mathrm{mL}\right)$, but did not infect the wild-type P. aeruginosa (Figure 3c). However, when Pf4r was expressed in the Pf4 deletion mutant, no plaque formation was observed. This suggests that Pf4r was sufficient to confer immunity against phage infection, in agreement with the work of Li et al. [21]. Unexpectedly, there was also no plaque formation for the complemented strain in the absence of arabinose induction. Therefore, $p f 4 r$ expression was assessed by qRT-PCR (Figure S3). The copy number of $p f 4 r$ was $7.22 \times 10^{7}$ for arabinose-induced PAO1 $\Delta$ Pf4 pPf4r and $1.54 \times 10^{5}$ without arabinose induction, much higher than was in the wild type $\left(4.77 \times 10^{4}\right)$. Thus, the arabinose expression system is leaky, even in the absence of the inducer, evidently due to the difference in catabolite repression in P. aeruginosa as compared to E. coli, and this is in agreement with previously published work [49]. Therefore, for the remainder of this work, arabinose was not added to any of the cultures. In contrast, for the PAO1 $\triangle \mathrm{Pf} 4$ strains complemented with the pPf4r containing mutation(s), there was a loss in immunity for the $\mathrm{pPf} 4 \mathrm{r}_{\mathrm{A}>\mathrm{G}}\left(1.1 \times 10^{6} \mathrm{PFU} / \mathrm{mL}\right)$ and the $\mathrm{pPf} \mathrm{r}^{*}\left(1.15 \times 10^{6} \mathrm{PFU} / \mathrm{mL}\right)$, 
while the $\mathrm{pPf} \mathrm{r}_{\mathrm{C}>\mathrm{A}}$ complementation remained immune. This loss of function could be due to the loss of dimerisation of the homodimer.

We expect that the serine to proline mutation $(788799 \mathrm{~A}>\mathrm{G})$ may affect the binding constant of the homo-dimerisation. Therefore, to determine if Pf4r forms dimers and the effect of this mutation on protein dimerisation, we performed gel-filtration size exclusion chromatography (SEC) and multi-angle light scattering (MALS). This revealed that both Pf4r and Pf6r form dimers in biological buffers. In contrast, Pf4r* eluted as a monomer with a molecular mass equivalent of a dimer as determined by MALS (Figure 4). This would suggest that the mutations in Pf4r* significantly reduce dimerisation and hence, prevent DNA binding.

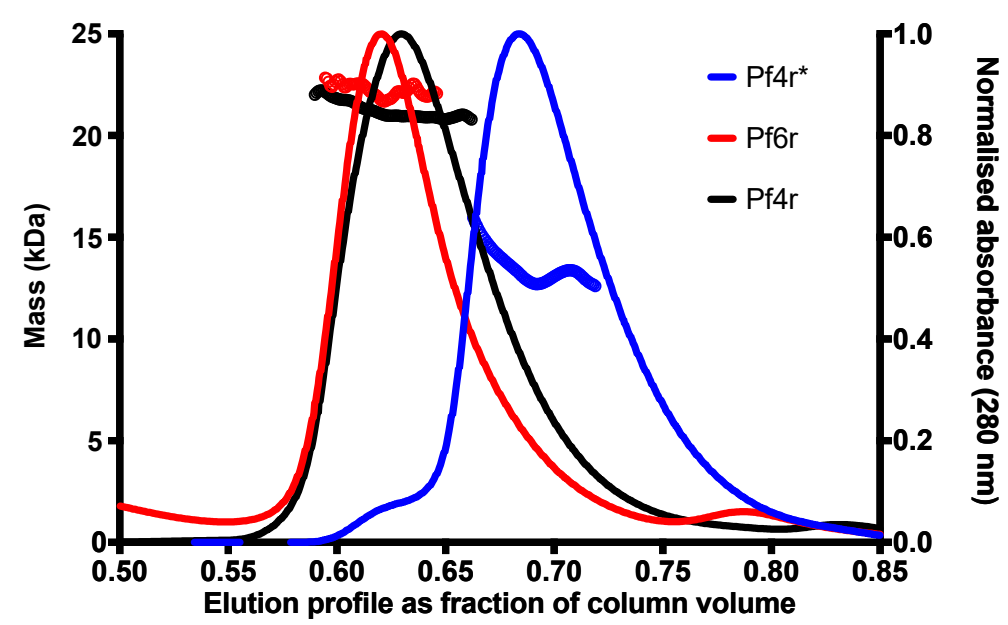

Figure 4. Pf4r (red), Pf4r* (blue) and Pf6r (black) elution profiles on SEC with molecular mass determination by MALS. Markers across the peaks represent the molar mass distribution (open circles, left axis, $\mathrm{kDa}$ ) of the protein species within the peak as determined in-line MALS (Pf4r $21 \mathrm{kDa}$, Pf4r* $13.7 \mathrm{kDa}$ and Pf6r $21.9 \mathrm{kDa}$ ). Theoretical masses of Pf4r and Pf4r* are $10.5 \mathrm{kDa}(21 \mathrm{kDa}$ dimer), and Pf6r is $11.2 \mathrm{kDa}$ (dimer $22.4 \mathrm{kDa}$ ).

\subsection{Mutations in the Promoter Regions and in Pf4r Affects DNA-Protein Binding}

The pf4r gene is localized in the intergenic region of PA0716 and PA0717 $(788,542-788,808)[21,45]$. Mutations were found in both the $p f 4 r$ coding region $(788570 \mathrm{C}>\mathrm{A}$ and 788799A $>$ G) as well as upstream of the ORF $(788826 \mathrm{G}>\mathrm{T}$ and 788857A $>\mathrm{G})$ in SI isolates of $P$. aeruginosa [45] (Figure 5a). The position of the mutations in the $5^{\prime}$ region and as shown by Li et al. [21] suggests that mutations in the promoter region of $p f 4 r$, referred to here as $\mathrm{P}_{p f 4 r}$ (to indicate the wild-type promoter), would affect Pf4r binding. The SI infective phage carried mutations in this region as well as non-synonymous mutations within the $p f 4 r$ gene (Figure 5a), suggesting that SI could be due to the failure of the wild-type Pf4r to bind to the altered promoter region. Therefore, $\mathrm{P}_{p f 4 r-\mathrm{G}>\mathrm{T}}, \mathrm{P}_{p f 4 r-\mathrm{A}>\mathrm{G}}$ and $\mathrm{P}_{p f 4 r \text {-doubleSNP }}$ probes which contained the mutations in the putative promoter region previously observed [45] and the mutated protein, Pf4r*, were tested using in vitro binding assays (EMSA) (Figure 5b). As expected and confirmatory of what was demonstrated by Li et al. [21], the $\mathrm{P}_{p f 4 r}$ probe showed slower migration after incubation with the wild-type Pf4r (Figure 5b-i) at $20 \mathrm{nM}$ and showed two distinct bands that suggested the presence of both single (C1) and putative dimer or multimer protein-DNA complexes (C2). Only the dimer or multimer proteinDNA complex was observed at $80 \mathrm{nM}$ of Pf4r. In contrast to the wild-type promoter sequence $\left(\mathrm{P}_{p f 4 r}\right)$, the formation of a protein-DNA complex was observed only at $\geq 70 \mathrm{nM}$ (Figure 5b-ii) for the $\mathrm{P}_{p f 4 r-\mathrm{G}>\mathrm{T}}$ probe, while there was no binding to both the $\mathrm{P}_{p f 4 r-\mathrm{A}>\mathrm{G}}$ and

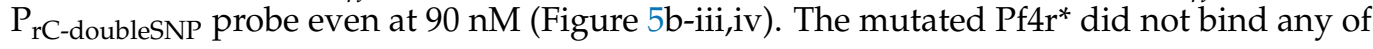
the DNA targets used (Figure $5 \mathrm{~b}-\mathrm{vi}-\mathrm{x}$ ). 
(a)

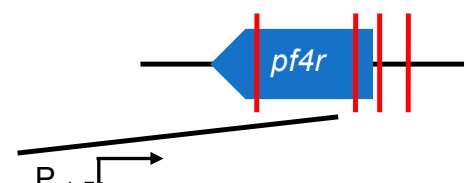

GCT ACGTGCTCA I ATAGAAATTTATTCCGGGGAAATAGTTGCCCTGACGTGGCTT

$788799 \mathrm{~A}>\overline{\mathrm{G}}$ (Ser4Pro)

DR IR

$7888 \overline{2} 6 \mathrm{G}>\mathrm{T}$

TGGGGAАATAАTTTCTCCAGGGTAАTTATTTCTCTAGCTGCGTATGGCTAATGCCA

$7888 \overline{5} 7 \mathrm{~A}>\mathrm{G}$

(b)

DNA targets

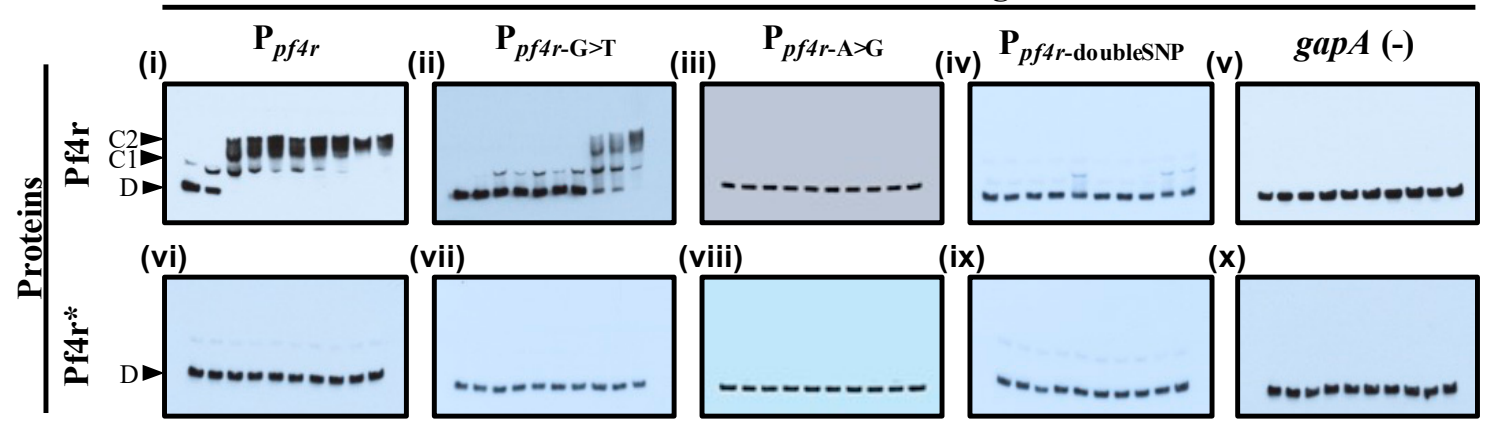

Figure 5. EMSA of Pf4r and Pf4r* protein gradients on target DNA. (a) The positions of $p f 4 r$ relative to the Pf4 genes and the single nucleotide polymorphisms within the $p f 4 r$ region relative to the PAO1 genome are indicated (red vertical lines). Italicised nucleotide sequences are part of the probe used in the EMSA. The locations of the direct repeats (DR), inverted repeats (IR) and promoter start sites were as seen in Li et al. [21]. (b) $\mathrm{P}_{p f 4 r}$ indicates the wild-type promoter region. The $\mathrm{P}_{p f 4 r-\mathrm{A}>\mathrm{G}}, \mathrm{P}_{p f 4 r-\mathrm{G}>\mathrm{T}}$ and $\mathrm{P}_{p f 4 r \text {-doubleSNP }}$ probes have mutations in either 788857A>G or 788826G>T, or both positions, respectively. The binding of Pf4r and Pf4r* to the selected target regions was tested using an increasing protein gradient (lanes 1-10 were 0, 10, 20, 30, 40, 50, 60, 70, 80 and $90 \mathrm{nM}$, respectively). The non-target gap $A$ (100 bp) gene was randomly chosen as the negative control for the assays. The notations indicate free DNA (D), protein-DNA complex (C1) and protein dimer-DNA complex (C2). Pf4r restores LasB activity to the Pf4 knockout and increases frequency of variants.

In addition to regulating immunity against infection, we wondered if the Pf4r had additional roles in PAO1. Previous observations showed that the Pf4 phage was associated with increased virulence and biofilm formation by P. aeruginosa PAO1 [13]. This suggests that the Pf4r may also control host gene expression. We first assayed if Pf4r had an effect on the expression levels of some common virulence factors such as iron chelation as well as secreted proteases [50]. For this experiment, biofilms of wild-type P. aeruginosa PAO1, the Pf4 deletion mutant, as well as the Pf4 deletion mutant complemented with either pPf4r or pPf4r* were grown for 2 days, and their effluent was collected daily. In biofilms, Pf4r restored the LasB activity of the Pf4 deletion mutant to wild-type levels by the second day of biofilm growth (Figure 6c). The pyocyanin levels were lower in the Pf4 deletion mutant but were restored by both Pf4r and Pf4r* complementation on day 1, although there were not many differences on day 2 (Figure $6 \mathrm{~b}$ ). In the case of pyoverdine, the Pf 4 deletion mutant overproduces pyoverdine on both days, but complementation with Pf4r and Pf4r* has no effect on pyoverdine levels (Figure 6c).

Mismatch repair and reactive oxygen and nitrogen species are linked to increased production of morphotypic variants, which are linked mutations in the host genome [14]. To determine if Pf4r contributed to an increase in variant formation, Pf4r and Pf4r* was complemented in both the $\Delta \mathrm{Pf} 4$ and the wild-type strains. There was no significant difference in the survival rates between the strains after 30 min of $\mathrm{H}_{2} \mathrm{O}_{2}$ treatment (Figure $6 \mathrm{~d}$ ). In contrast, while not a statistically significant difference, the percentage of variants induced by $\mathrm{H}_{2} \mathrm{O}_{2}$ was slightly higher when Pf4r was expressed in both the wild type and Pf4 mutant strains (e.g., $50 \mathrm{mM} \mathrm{H} \mathrm{O}_{2}: 16.3 \% \pm 2.5 \%$ for $\Delta \mathrm{Pf} 4 \mathrm{pPf} 4 \mathrm{r}, 13.3 \% \pm 1.6 \%$ for $\Delta \mathrm{Pf} 4$, 
$19.9 \% \pm 0.7 \%$ for PAO1 pPf4r and $17.5 \% \pm 2.3 \%$ for PAO1) (Figure $6 e$ ). This increase in variants was not observed when the Pf4r* construct was introduced into these strains.

(a)

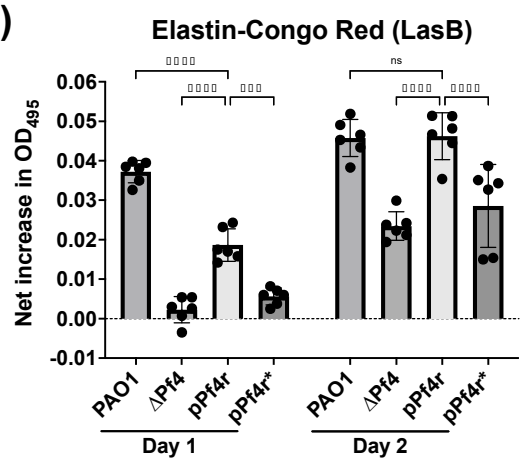

(d)

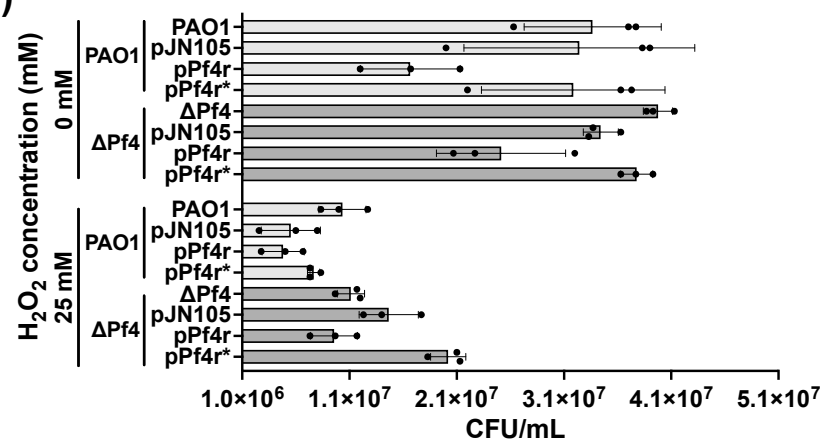

(b)

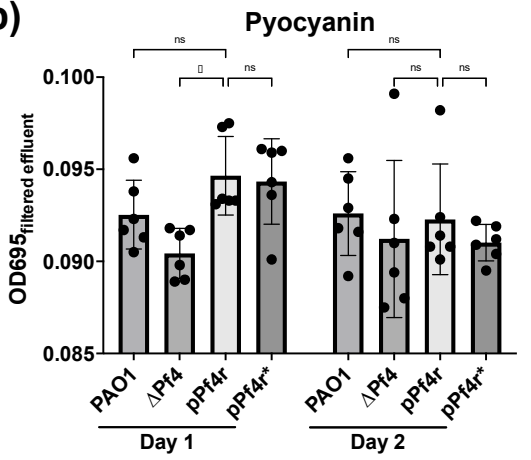

(c)

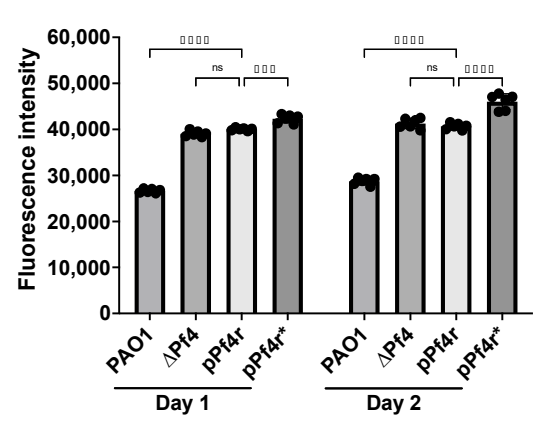

(e)

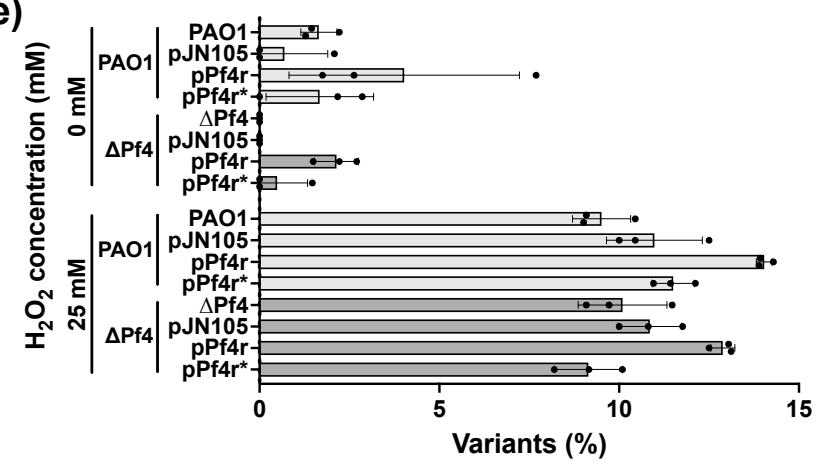

Figure 6. Pf4r restores LasB activity and slightly increases mutant frequencies. (a,b) Pyoverdine and pyocyanin production were semi-quantified by measuring emission fluorescence at $460 \mathrm{~nm}$ and by absorbance at $\mathrm{OD}_{695}$, respectively. (c) Extracellular protease activity in cell-free supernatant was measured using elastin-congo red for LasB (increase in absorbance at $\mathrm{OD}_{495}$ ). Significant difference was determined using Dunnett's multiple comparisons test. (d) The number of viable cells after $25 \mathrm{mM} \mathrm{H}_{2} \mathrm{O}_{2}$ treatment for $30 \mathrm{~min}$ and (e) percentage of the mutant frequencies of $\triangle \mathrm{Pf} 4$ and PAO1 after treatment with $\mathrm{H}_{2} \mathrm{O}_{2}$. For all assays, $N=3$.

\subsection{Pf4r Can Bind and Regulate Pseudomonas Genes}

Based on the observation that Pf4r confers immunity against reinfection as well as affects LasB and pyoverdine expression as well as variant formation, we investigated whether Pf4r affects other P. aeruginosa genes. Therefore, to determine if Pf4r also functions more generally as a transcriptional regulator in P. aeruginosa, ChIPseq was performed with both Pf4r and Pf4r*. Based on the peaks detected by ChIPseq, a binding motif (SSRGGGCAAYADYTTCCTSGH) was predicted and subsequently used to scan the genome to find the potential regulon of the proteins.

No detectable DNA was obtained for the mutant Pf4r*. This is in agreement with the observation that Pf4r* did not bind any of the DNA targets (Figure $5 b$ ) due to the failure to dimerise (Figure 4). For the wild-type Pf4r, 46 peaks were detected, suggesting the protein binds to those genes (Figure 7a and Table S4), with one of the binding sites being the $\mathrm{P}_{p f 4 r}$ (i.e., as predicted based on the DNA binding data), here labelled as the intergenic region of PA0717 (peak region 787960-790399) (Figure 7a and Table 1). 
(a)

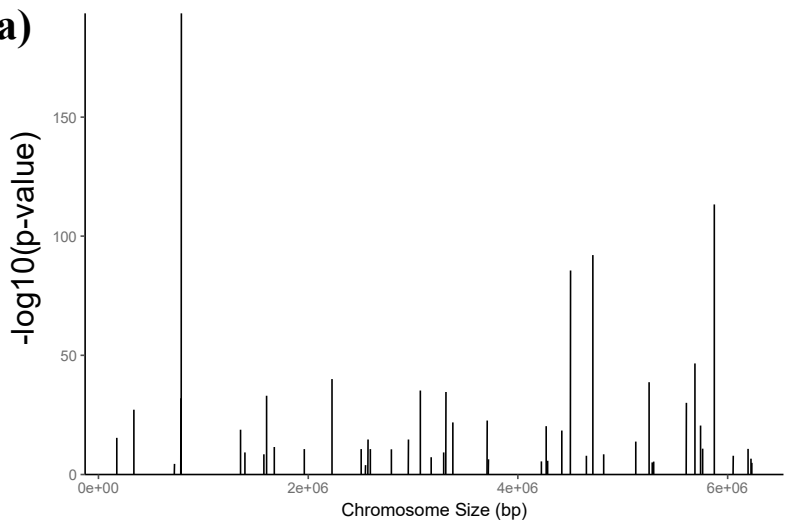

(b)

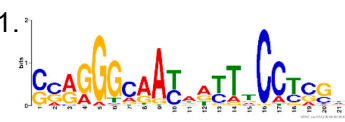

2.

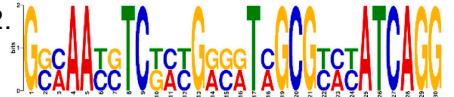

3. ${ }^{2}$

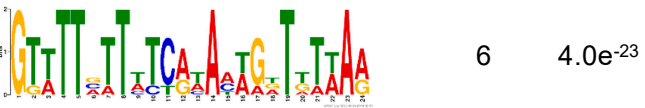

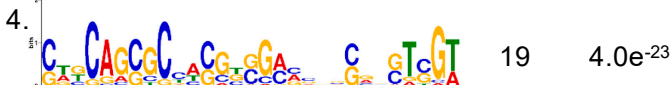

(c)

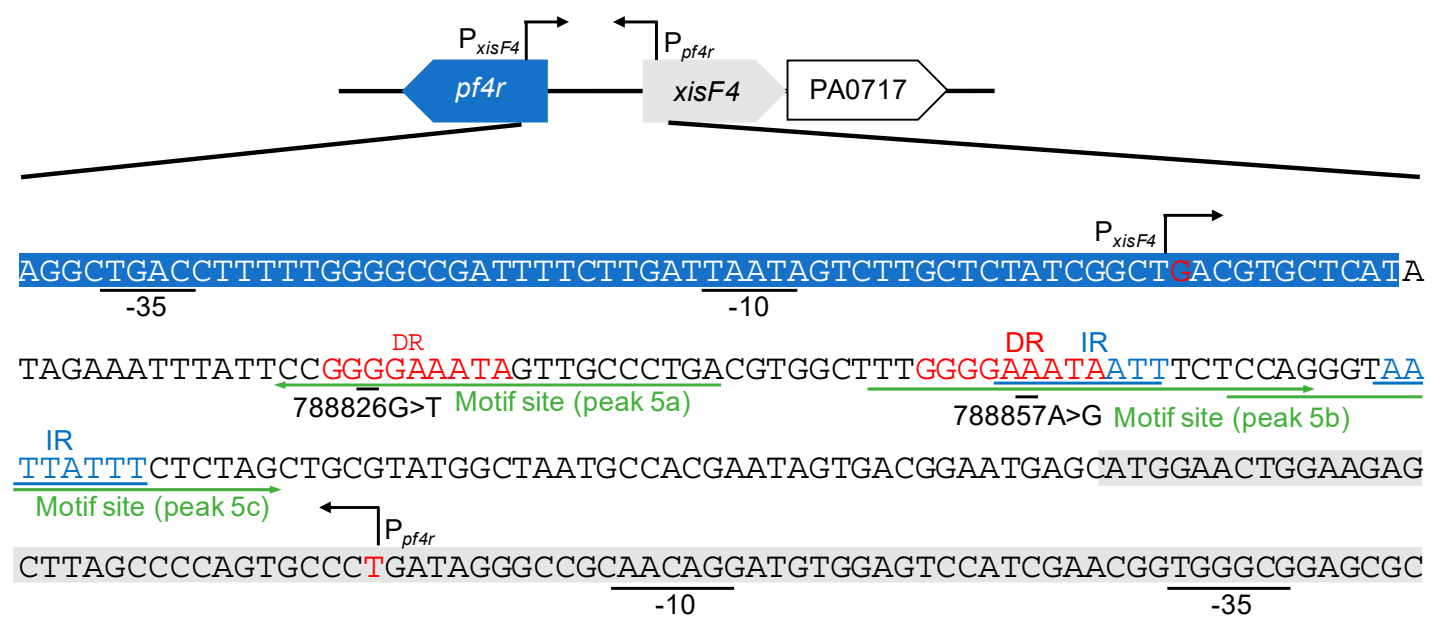

Figure 7. Peak coverage plot and predicted binding motifs of Pf4r. (a) The relative positions of the detected peaks bound by Pf4r in the PAO1 genome. The peaks' heights were the - $\log 10$ ( $p$ value) obtained from MACS2 and represent the probability of the peak being a true match. (b) For every motif, the measurement of conservation is represented as a bit score (y-axis) for every nucleotide in their respective position ( $x$-axis). The occurrence of the motif in the submitted sequences is represented as 'Sites'. (c) Schematic diagram of the $p f 4 r$ and $x i s F 4$ intergenic region. The locations of the $-10,-35$, direct repeats (DR), inverted repeats (IR) and promoter start sites were as seen in Li et al. [21]. The Pf4r binding motifs as found by VirtualFootprint are underlined in green, found in the negative strand indicated by a left-facing arrow or in the positive strand when indicated by a right-facing arrow.

To identify other potential regulons of Pf4r not detected by ChIPseq analysis, a putative binding motif was obtained using MEME-ChIP [40] from the sequences associated with the ChIPseq peaks. Four possible motifs were obtained, ranked by their E-value (Figure $7 \mathrm{~b}$ ). In the subsequent analysis, only the top-scoring motif, SSRGGGCAAYADYTTCCTSGH (motif 1), was used, which was found in 32 of the 46 regions identified in the ChIPseq dataset (Table S5).

An additional 853 potential binding regions were detected by Virtual Footprint using the top-scoring motif. Of those, 87 were located in intergenic regions and 766 within coding regions (Table S6). A consensus table of ChIPseq peaks and Virtual Footprint matches was generated to assist with the analysis (Table 1). As expected, one of the genes detected by the Virtual Footprint analysis was the $p f 4 r$ putative promoter region and was also detected as one of the ChIPseq peaks (Table 1, peaks 5a-c). Other genes observed in the ChIPseq dataset, $p h z A 1$ and pilQ, which are associated with virulence, were also identified as part of the virtual regulon of Pf4r. The potential targets for regulation by the Pf4r include several other virulence-factor related genes such as tle5b (a T6SS effector) and $\operatorname{apr} A$ (a precursor of alkaline metalloproteinase), as well as genes associated with energy and central metabolisms such as fix $\mathrm{C}$ (an oxidoreductase) and $a m b A$ (a putative LysE-type translocator). 
Table 1. Consensus ChIPseq peaks detected and predicted gene targets of the Pf4r.

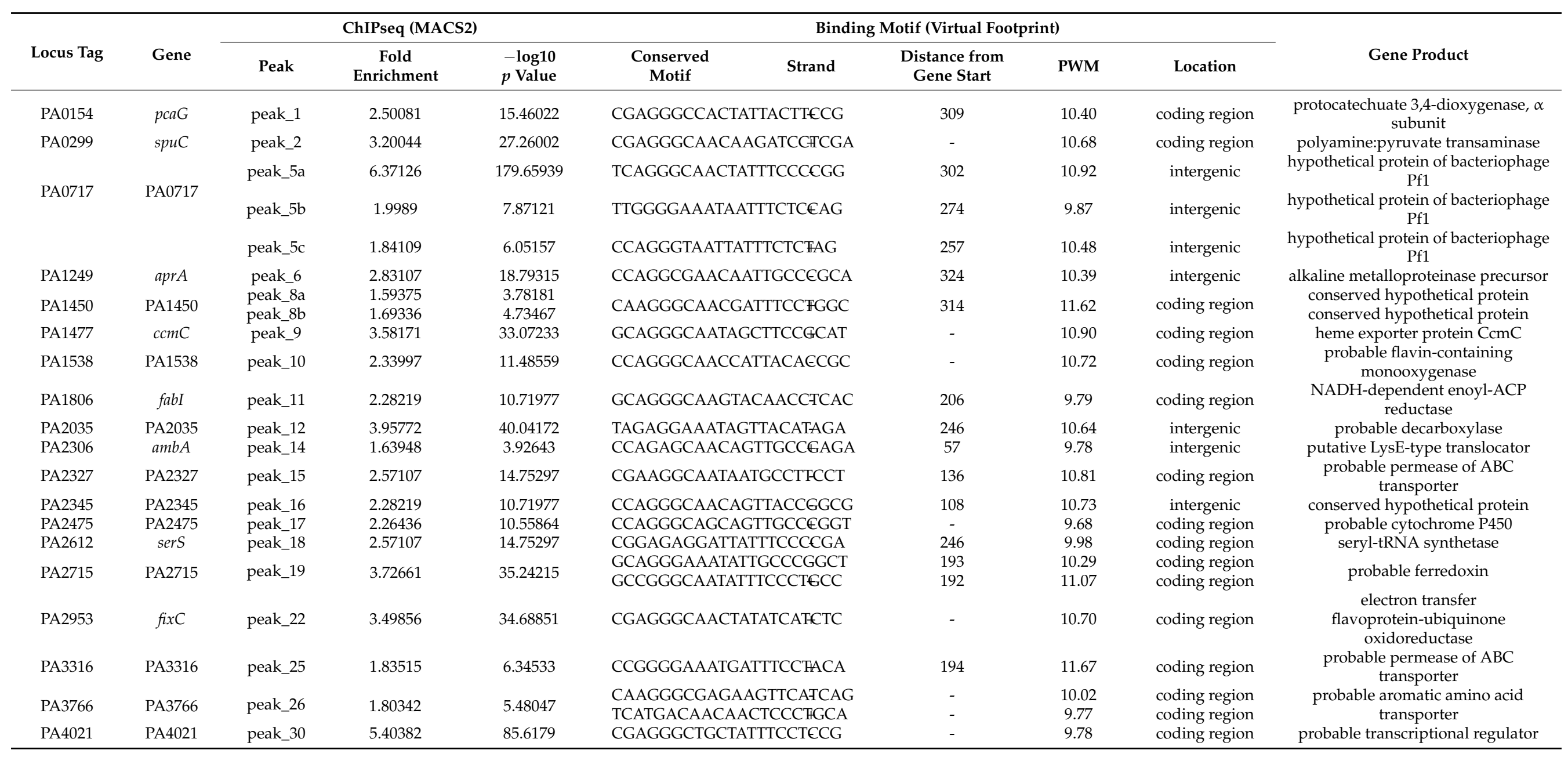


Table 1. Cont.

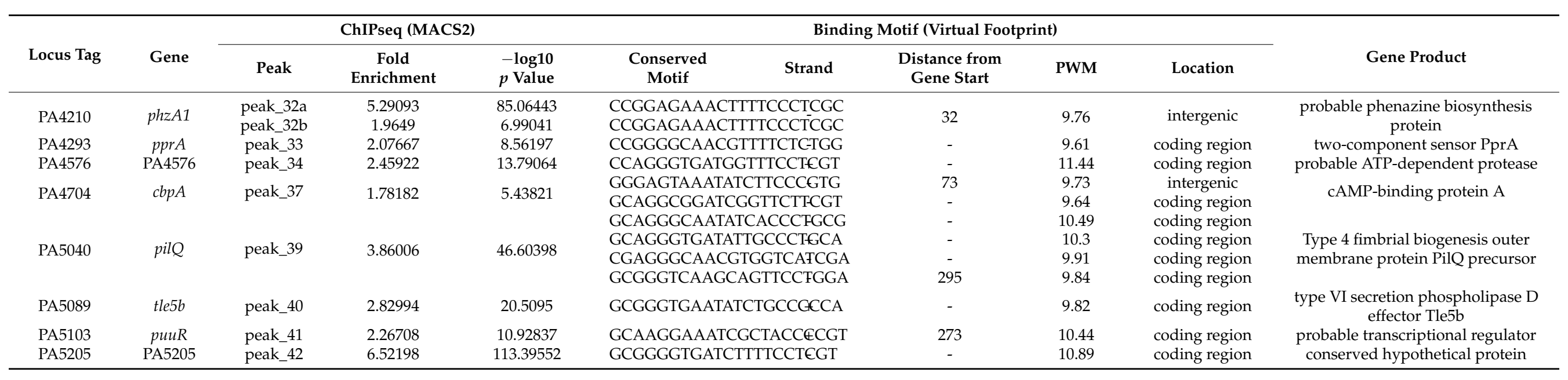


The ChIPseq analysis suggests that Pf4r controls P. aeruginosa PAO1 gene expression. To further explore the effect of Pf4r on P. aeruginosa gene expression, changes in global gene expression were determined. For this analysis, the wild-type P. aeruginosa and the deletion mutant complemented with the wild-type $p f 4 r$ were compared relative to the Pf4 deletion mutant. As Pf4r* was not able to bind to any of the DNA targets during EMSA (Figure 5) and during DNA pulldown for ChIPseq, complementation with Pf4r* was not completed for RNAseq. As expected, the samples for the wild type and PAO1 $\Delta$ Pf4 mutant clustered separately, while PAO1 $\Delta \mathrm{Pf} 4 \mathrm{pPf} 4 \mathrm{r}$ also clustered away from the PAO1 $\Delta \mathrm{Pf} 4$ mutant (Figure S4a), suggesting altered expression by complementation of wild-type Pf4r.

Similarities in the differentially expressed genes (irrespective of whether they were induced or repressed) were compared between the Pf4r complemented strain as well as the wild-type P. aeruginosa strain relative to the Pf4 deletion mutant (Table S9 and Figure S4). This was to determine if the differences in gene expression of the wild type could be attributed to the presence of Pf4r to explain the reported phenotypic differences observed for the wild-type P. aeruginosa and the Pf4 deletion mutant (e.g., increased virulence and resistance to SDS) [13]. Several genes were upregulated across all of the comparisons, including PA2698 and PA1202, which are probable hydrolases, hypothetical protein PA2699 and $c d r A$ (a cyclic diguanylate-regulated TPS partner). Genes that were identified in both the RNAseq and Virtual Footprint data sets (Table S12) included aprA, gapN and mexF, while $c d r A$ was also found in the ChIPseq data set as well as the RNAseq and Virtual Footprint data sets.

\section{Discussion}

The filamentous Pf4 phage has been shown to drive multiple biofilm phenotypes of its host, $P$. aeruginosa, including cell death, colony expansion, stability of the biofilm and morphotypic variant formation. The Pf4 phage is also associated with increased virulence in a mouse infection model [13], and it has recently been shown that there is a positive correlation between the presence of Pf phage and chronic lung infection [16]. These phenotypes are further linked to the conversion of the phage into a form that can reinfect the host, causing cell death. Here, we have shown through molecular methods and phenotypic assays how the Pf4 phage plays a role in aspects of the biofilm mode of growth through regulation of host gene expression, in addition to the previously demonstrated physical contribution of phage particles to the properties of the biofilm matrix $[15,17]$.

\subsection{Pf4r Binds to Conserved Promoter Sites as a Dimer}

Pf4r shares homology with the E. coli phage P2 repressor C protein [17], and the P2 repressor $C$ play a role in the lytic and lysogenic switch [51,52]. Central to these effects of the Pf4 phage in P. aeruginosa is the repressor protein, Pf4r, which not only controls immunity to infection and phage replication [21] but also acts to regulate the expression of host genes. Here, we demonstrated that the protein functions as a dimer to bind the target promoters.

The data are supportive data that the upstream region of Pf4r is the promoter of the $p f 4 r$ gene as ChIPseq pulled down this region (Table 1). Additionally, the Pf4r binding motif was found in three sites of the region that overlapped with the direct and inverted repeats previously reported (Figure 7c) [21]. Mutations within this region were observed by Hui et al. [14] and McElroy et al. [45], and it was demonstrated here that the binding of Pf4r to that mutated promoter region was altered. It was also observed that there is a mutation in both binding sites of Pf4r, in the highly conserved guanine residue of the conserved motif $(788826 \mathrm{G}>\mathrm{T}$, Figure $5 \mathrm{a}$; motif site $5 \mathrm{a}$, Figure $7 \mathrm{c}$ ) and in a relatively conserved adenine residue (788857A $>\mathrm{G}$, Figure 5a; motif site 5b, Figure 7c) [14,45]. The experimental data here showed that a single mutation could reduce Pf4r binding while mutations in the two sites completely abolished binding (Figure 5).

The Pf4r shows the closest homology to the repressor C protein of the temperate E. coli phage $\mathrm{P} 2$. The repressor $\mathrm{C}$ protein is a DNA-binding protein that forms a symmetric dimer, 
allowing it to bind non-palindromic direct DNA repeats via two helices (one located in each monomer) [47]. The helices are oriented in such a way that they can bind the major groove over two turns of the DNA. In support of this, the gel filtration and MALS result show that the Pf4r elutes as dimers and mutations arising in Pf4r* affect its ability to either fold or/and dimerise, causing its elution as a monomer. This would also explain why the mutant Pf4r* does not bind at all, even to the altered promoter region (Figure 5b), despite these mutations co-occurring in the superinfective Pf4-producing PAO1 morphotype [14,45]. The mutation in the dimerisation site (Figure 3a) also affected Pf4r's function as an immunity protein as the 788799A $>$ G (Ser4Pro) removed the host immunity to Pf4 infection while the 788570C $>A$ (Arg80Leu) does not (Figure 3c).

\subsection{Pf4r Mediated Regulation of Host Genes Associated with Biofilm Development, Virulence and Mediating Superinfection}

Since Pf4r is a DNA-binding protein and is important for immunity against Pf4 reinfection, we hypothesised that complementing Pf4r in a Pf4 mutant would restore some of the strain's phenotype to that of the wild type. Complementation was observed for LasB and pyocyanin activity in biofilms (Figure 6a-b). To assay for the global regulon affected by the Pf4r protein, ChIPseq and RNAseq were performed. Pf4r appears to regulate the expression of virulence factors, including pldB (Tables S4 and S6), extracellular proteases and siderophores (Table 1, Tables S4, S6 and S9). Based on the RNAseq data for the Pf4r-complemented PAO1 $\Delta$ Pf4, genes for intracellular proteases clpP2 and $p f p I$ were downregulated (Table S11). These genes were previously shown to affect biofilm formation in P. aeruginosa [53]. While the results suggest that Pf4r plays a role in regulating the expression of these virulence factors, more work is needed to more clearly elucidate the mechanism of this regulation. For example, does Pf4r induce or repress virulence gene expression after binding to the promoter regions, or does Pf4r interact with the transcriptional regulators for these virulence genes?

It was further previously reported that biofilms of $P$. aeruginosa generate morphotypic variants at the same time the superinfective phage variant is observed [14] and that such variants are linked to mutations in the P. aeruginosa genome [33]. It is possible that in PAO1 biofilms, the appearance of variants is partly controlled Pf4r through repression of $p f p I$, which is involved in general stress protection [35]. Similarly, many of these morphotypic variants also produce SI Pf4 phages [14,45]. The SI Pf4 could cause the lysis of the wild-type strain, increasing the genetic diversity and overall fitness of the biofilm. Indeed, we have observed that overexpression of Pf4r, either in the phage deletion mutant or the wild-type $P$. aeruginosa, is associated with increased variant formation. It is therefore hypothesised that Pf4r represses expression of $p f p I$, which subsequently inhibits the general stress protection response, allowing for mutations to occur that lead to increased numbers of morphotypic variants as well as the superinfective variants. Further work is needed to explore the relationship between Pf4r, pfpI and the mechanism of variant formation.

The data presented here as well as in previous studies [14,21,45] provide an insight into the possible mechanism of how the Pf4 phage is able to cause superinfection (SI) in $P$. aeruginosa, which is otherwise immune to Pf4 reinfection due to the presence of the Pf4 prophage within its genome. The normally expressed and released filamentous phage do not cause cell lysis. In the case of superinfection, it is possible that lysis is due to uncontrolled phage production, which overtaxes the membrane as the phage particles were released. As recently shown, an increase in Pf4 titres was due to a lack of repression of XisF4, which in turn positively regulates PA0727 (phage-initiator gene) [21]. This mechanism is similar to the $E$. coli $\lambda$ phage, where mutations within the $c I$ promoter disrupt repressor binding and induce the lytic life cycle of the phage [54,55]. As with Pf4, Pf6 also causes superinfection in P. aeruginosa but only from day 6 of biofilm growth. The plaques observed on day 6 are likely to be SI Pf6, based on the qPCR data; however, this could be further confirmed in the future by either purification and recovery of the phage or direct sequencing. As for Pf4, we hypothesise that similar mechanisms of gene regulation are 
involved for Pf6. However, due to the lack of a Pf6 deletion mutant, similar molecular and phenotypic assays could not be performed.

Filamentous phage clearly play important roles in other bacterial species, in particular in the context of association with a eukaryotic host. For example, the RSS1 phage of Ralstonia has been shown to increase bacterial virulence through increased polysaccharide production and virulence expression [56]. Other filamentous phage also have transcriptional repressors within their genomes. The cf phage of Xanthomonas campestris pv. citri has a hypothetical repressor protein that can interfere with RNA-RNA and DNA-protein interactions which regulate lysogeny [57]. In the $V$. cholerae CTX $\Phi$ phage, the RstR protein represses the $r s t A$ promoter by binding as a dimer-of-dimers and regulates phage replication $[58,59]$. Given that these filamentous phage have different regulatory genes, despite having otherwise conserved core genes, it is suggested that the regulatory functions have been acquired independently by each of the phage and hence may infer that they regulate immunity slightly differently and that they may also interact with the host in different ways, e.g., in terms of host gene regulation. As shown here, Pf4r plays a role in regulating a number of biofilm- and virulence-related genes, and those correlate with defects in biofilm development and virulence in vivo. Given the broader distribution of filamentous phage in bacteria and the conservation of the repressor protein $[10,60]$, it is likely that filamentous phages are also important in regulating functions related to biofilm formation and host colonisation by those bacteria as well.

\section{Conclusions}

The data presented in this study suggest that the Pf4r is not only important in the control of superinfection but may also be an important regulator of virulence-factor-related genes. The Pf4r binds its putative promoter as a homodimer and regulates the expression of Pf4 genes. Mutations in the Pf4r at the dimer interface (788799A $>$ G; Ser $>$ Pro) prevented dimerisation from disrupting its function as a transcriptional regulator. The data here suggest that Pf4r contributes to the accumulation of mutations in the genome of PAO1, as well as mutations in Pf4r and its promoter region, converting Pf4 to its superinfective form. Given that filamentous phage have recently been reported in a broad range of bacteria and archaea from almost all habitats, it is possible that these phage play an important role in the regulation of biofilm and virulence responses of their hosts. This work highlights that simple genetic elements, such as the filamentous phage, can play a significant, albeit complex, role in the lifestyle and function of the host bacterium.

Supplementary Materials: The following are available online at https:/ / www.mdpi.com/article/10 $.3390 / v 13081614$ /s1, Figure S1. Sequence of site-directed mutagenesis and region of EMSA probe. Figure S2. Crystal structure of Pf6r. Figure S3. Leaky expression of $p f 4 r$ with the araBAD-promoter system. Figure S4. Ordination of complemented strains against the wild type and Pf4 knockout, and comparison of top 20 differentially expressed genes. Table S1. Bacterial strains and plasmids used in this study. Table S2. List of primers used. Table S3. Crystallography data collection and refinement. Table S4. ChIPseq peaks for Pf4r. Table S5. Sequences for Pf4r binding motif 1. Table S6. Pf4r binding regions detected by Virtual Footprint. Table S7. Test for the effect of complementation on gene expression. Table S8. Permutation test for homogeneity of multivariate dispersion. Table S9. Common differentially expressed genes relative to the $\Delta \mathrm{Pf} 4$ mutant. Table S10. Genes differentially regulated in PAO1 $\triangle \mathrm{Pf} 4$ biofilms relative to wild-type P. aeruginosa. Table S11. Genes differentially regulated in PAO1 $\triangle \mathrm{Pf} 4 \mathrm{pPf} 4 \mathrm{r}$ biofilms relative to PAO1 $\Delta \mathrm{Pf} 4$. Table S12. Genes common in RNAseq and Virtual Footprint.

Author Contributions: M.H.I. and S.A.R. designed the study; K.A.M., P.N. and I.G.D. performed the crystallisation studies; M.H.I. and Y.F.G. performed all other experiments. All authors, including D.M. and S.K., contributed to the data analysis and drafting of the manuscript. All authors have read and agreed to the published version of the manuscript. 
Funding: This research was supported by the Singapore Centre for Environmental Life Sciences Engineering (SCELSE), whose research is supported by the National Research Foundation Singapore, Ministry of Education, Nanyang Technological University and the National University of Singapore, under its Research Centre of Excellence Programme. MH Ismail was supported by the National Research Foundation Singapore under its National Research Foundation (NRF) Environmental and Water Technologies (EWT) PhD Scholarship Programme, administered by the Environment and Water Industry Programme Office (EWI). The structural studies were financially supported by the Australian Research Council LIEF Funding Scheme (LE190100165) for contributions towards the Structural Biology Facility within the Mark Wainwright Analytical Centre at the University of New South Wales.

Institutional Review Board Statement: Not applicable.

Informed Consent Statement: Not applicable.

Data Availability Statement: All sequencing data that support the findings of this study have been deposited in the NCBI Gene Expression Omnibus (GEO), accessible through the GEO series accession number GSE154459. The crystallographic data have been deposited in the Protein Data Bank (PDB) with the PDB IDs 6WNM and 6WPZ for Pf4r* and 6X6F for Pf6r. All other relevant data are available from the corresponding author upon request.

Acknowledgments: We thank the NTU Protein Production Platform (www.proteins.sg) for the cloning, expression tests and purification of protein constructs Pf4r, Pf4r* and Pf6r. Parts of this manuscript was part of the doctoral thesis of Muhammad Hafiz Ismail in Nanyang Technological University. We acknowledge the Structural Biology Facility Mark Wainwright Analytical Centre at the University of New South Wales for X-ray crystallography support. This research was undertaken on the MX1 and MX2 beamline at the Australian Synchrotron, part of ANSTO.

Conflicts of Interest: The authors declare no conflict of interest.

\section{References}

1. Holmes, R.K. Biology and Molecular Epidemiology of Diphtheria Toxin and the tox Gene. J. Infect. Dis. 2000, 181, S156-S167. [CrossRef] [PubMed]

2. Rodríguez-Rubio, L.; Haarmann, N.; Schwidder, M.; Muniesa, M.; Schmidt, H. Bacteriophages of Shiga Toxin-Producing Escherichia coli and Their Contribution to Pathogenicity. Pathogens 2021, 10, 404. [CrossRef]

3. Wagner, P.L.; Neely, M.N.; Zhang, X.; Acheson, D.W.K.; Waldor, M.K.; Friedman, D. Role for a Phage Promoter in Shiga Toxin 2 Expression from a Pathogenic Escherichia coli Strain. J. Bacteriol. 2001, 183, 2081-2085. [CrossRef] [PubMed]

4. Boles, B.R.; Singh, P.K. Endogenous oxidative stress produces diversity and adaptability in biofilm communities. Proc. Natl. Acad. Sci. USA 2008, 105, 12503-12508. [CrossRef]

5. Farrant, J.L.; Sansone, A.; Canvin, J.R.; Pallen, M.J.; Langford, P.R.; Wallis, T.S.; Dougan, G.; Kroll, J.S. Bacterial copper- and zinc-cofactored superoxide dismutase contributes to the pathogenesis of systemic salmonellosis. Mol. Microbiol. 1997, 25, 785-796. [CrossRef] [PubMed]

6. $\quad$ Lim, W.S.; Ephang, K.K.S.; Etan, A.H.-M.; Eli, S.F.-Y.; Ow, D.S.-W. Small Colony Variants and Single Nucleotide Variations in Pf1 Region of PB1 Phage-Resistant Pseudomonas aeruginosa. Front. Microbiol. 2016, 7, 282. [CrossRef] [PubMed]

7. Addy, H.S.; Askora, A.; Kawasaki, T.; Fujie, M.; Yamada, T. Loss of Virulence of the Phytopathogen Ralstonia solanacearum Through Infection by $\varphi$ RSM Filamentous Phages. Phytopathology 2012, 102, 469-477. [CrossRef] [PubMed]

8. Argov, T.; Azulay, G.; Pasechnek, A.; Stadnyuk, O.; Sapir, S.R.; Borovok, I.; Sigal, N.; Herskovits, A.A. Temperate bacteriophages as regulators of host behavior. Curr. Opin. Microbiol. 2017, 38, 81-87. [CrossRef] [PubMed]

9. Dion, M.B.; Oechslin, F.; Moineau, S. Phage diversity, genomics and phylogeny. Nat. Rev. Genet. 2020, 18, 125-138. [CrossRef] [PubMed]

10. Roux, S.; Krupovic, M.; Daly, R.A.; Borges, A.; Nayfach, S.; Schulz, F.; Sharrar, A.; Carnevali, P.B.M.; Cheng, J.-F.; Ivanova, N.N.; et al. Cryptic inoviruses revealed as pervasive in bacteria and archaea across Earth's biomes. Nat. Microbiol. 2019, 4, 1895-1906. [CrossRef]

11. Mai-Prochnow, A.; Hui, J.G.K.; Kjelleberg, S.; Rakonjac, J.; McDougald, D.; Rice, S.A. 'Big things in small packages: The genetics of filamentous phage and effects on fitness of their host'. FEMS Microbiol. Rev. 2015, 39, 465-487. [CrossRef] [PubMed]

12. Klockgether, J.; Munder, A.; Neugebauer, J.; Davenport, C.; Stanke, F.; Larbig, K.D.; Heeb, S.; Schöck, U.; Pohl, T.M.; Wiehlmann, L.; et al. Genome Diversity of Pseudomonas aeruginosa PAO1 Laboratory Strains. J. Bacteriol. 2010, 192, 1113-1121. [CrossRef] [PubMed]

13. Rice, S.A.; Tan, C.H.; Mikkelsen, P.J.; Kung, V.; Woo, J.; Tay, M.; Hauser, A.; McDougald, D.; Webb, J.; Kjelleberg, S. The biofilm life cycle and virulence of Pseudomonas aeruginosa are dependent on a filamentous prophage. ISME J. 2008, 3, 271-282. [CrossRef] [PubMed] 
14. Hui, J.G.K.; Mai-Prochnow, A.; Kjelleberg, S.; McDougald, D.; Rice, S.A. Environmental cues and genes involved in establishment of the superinfective Pf4 phage of Pseudomonas aeruginosa. Front. Microbiol. 2014, 5, 654. [CrossRef]

15. Secor, P.R.; Sweere, J.; Michaels, L.A.; Malkovskiy, A.V.; Lazzareschi, D.; Katznelson, E.; Rajadas, J.; Birnbaum, M.; Arrigoni, A.; Braun, K.R.; et al. Filamentous Bacteriophage Promote Biofilm Assembly and Function. Cell Host Microbe 2015, 18, 549-559. [CrossRef]

16. Secor, P.R.; Burgener, E.B.; Kinnersley, M.; Jennings, L.K.; Roman-Cruz, V.; Popescu, M.; Van Belleghem, J.D.; Haddock, N.; Copeland, C.; Michaels, L.A.; et al. Pf Bacteriophage and Their Impact on Pseudomonas Virulence, Mammalian Immunity, and Chronic Infections. Front. Immunol. 2020, 11, 244. [CrossRef]

17. Webb, J.; Lau, M.; Kjelleberg, S. Bacteriophage and Phenotypic Variation in Pseudomonas aeruginosa Biofilm Development. J. Bacteriol. 2004, 186, 8066-8073. [CrossRef]

18. Abedon, S.T. Bacteriophages and Biofilms. In Biofilms: Formation, Development and Properties; Nova Science Publishers: Hauppauge, NY, USA, 2011; p. 58.

19. Sweere, J.M.; Van Belleghem, J.D.; Ishak, H.; Bach, M.S.; Popescu, M.; Sunkari, V.; Kaber, G.; Manasherob, R.; Suh, G.A.; Cao, X.; et al. Bacteriophage trigger antiviral immunity and prevent clearance of bacterial infection. Science 2019, 363, eaat9691. [CrossRef]

20. Tay, M. The Role of Bacteriophage in Granulation; Nanyang Technological University: Singapore, 2013.

21. Li, Y.; Liu, X.; Tang, K.; Wang, P.; Zeng, Z.; Guo, Y.; Wang, X. Excisionase in Pf filamentous prophage controls lysis-lysogeny decision-making in Pseudomonas aeruginosa. Mol. Microbiol. 2018, 111, 495-513. [CrossRef]

22. Hernandez-Doria, J.D.; Sperandio, V. Bacteriophage Transcription Factor Cro Regulates Virulence Gene Expression in Enterohemorrhagic Escherichia coli. Cell Host Microbe 2018, 23, 607-617. [CrossRef]

23. Sambrook, J. Molecular Cloning: A Laboratory Manual, 3rd ed.; Cold Spring Harbor Laboratory Press: Hamlet in Long Island, NY, USA, 2001.

24. Ismail, M.H. The Role of Bacteriophages in Mixed Microbial Communities and Populations of Pseudomonas Aeruginosa; Nanyang Technological University: Singapore, 2019.

25. Some, D.; Amartely, H.; Tsadok, A.; Lebendiker, M. Characterization of Proteins by Size-Exclusion Chromatography Coupled to Multi-Angle Light Scattering (SEC-MALS). J. Vis. Exp. 2019, e59615. [CrossRef]

26. Liebschner, D. Protein Crystallography-Methods and Protocols. Acta Crystallogr. Sect. F Struct. Biol. Commun. 2018, 74, 74-75. [CrossRef]

27. Terwilliger, T.C.; Adams, P.; Read, R.; McCoy, A.J.; Moriarty, N.W.; Grosse-Kunstleve, R.W.; Afonine, P.V.; Zwart, P.H.; Hung, L.-W. Decision-making in structure solution using Bayesian estimates of map quality: The PHENIX AutoSolwizard. Acta Crystallogr. Sect. D Biol. Crystallogr. 2009, 65, 582-601. [CrossRef]

28. Liebschner, D.; Afonine, P.V.; Baker, M.L.; Bunkóczi, G.; Chen, V.B.; Croll, T.I.; Hintze, B.; Hung, L.-W.; Jain, S.; McCoy, A.J.; et al. Macromolecular structure determination using X-rays, neutrons and electrons: Recent developments in Phenix. Acta Crystallogr. Sect. D Struct. Biol. 2019, 75, 861-877. [CrossRef]

29. Emsley, P.; Lohkamp, B.; Scott, W.; Cowtan, K.D. Features and development of Coot. Acta Crystallogr. Sect. D Biol. Crystallogr. 2010, 66, 486-501. [CrossRef] [PubMed]

30. Bunkóczi, G.; Echols, N.; McCoy, A.J.; Oeffner, R.; Adams, P.; Read, R.J. Phaser. MRage: Automated molecular replacement. Acta Crystallogr. Sect. D Biol. Crystallogr. 2013, 69, 2276-2286. [CrossRef] [PubMed]

31. Krissinel, E.; Henrick, K. Protein Interfaces, Surfaces and Assemblies' Service PISA at European Bioinformatics Institute. 2007. Available online: http:/ / www.ebi.ac.uk/pdbe/prot_int/pistart.html (accessed on 17 July 2019).

32. Krissinel, E.; Henrick, K. Inference of Macromolecular Assemblies from Crystalline State. J. Mol. Biol. 2007, $372,774-797$. [CrossRef] [PubMed]

33. Lee, K.W.K.; Yam, J.K.H.; Mukherjee, M.; Periasamy, S.; Steinberg, P.D.; Kjelleberg, S.; Rice, S.A. Interspecific diversity reduces and functionally substitutes for intraspecific variation in biofilm communities. ISME J. 2015, 10, 846-857. [CrossRef]

34. Kessler, E.; Safrin, M. Elastinolytic and Proteolytic Enzymes. In Pseudomonas Methods and Protocols; Filloux, A., Ramos, J.-L., Eds.; Springer: New York, NY, USA, 2014; pp. 135-169.

35. Rodríguez-Rojas, A.; Blazquez, J. The Pseudomonas aeruginosa pfpI Gene Plays an Antimutator Role and Provides General Stress Protection. J. Bacteriol. 2009, 191, 844-850. [CrossRef]

36. Webb, J.S.; Thompson, L.S.; James, S.; Charlton, T.; Tolker-Nielsen, T.; Koch, B.; Givskov, M.; Kjelleberg, S. Cell Death in Pseudomonas aeruginosa Biofilm Development. J. Bacteriol. 2003, 185, 4585-4592. [CrossRef] [PubMed]

37. Bonocora, R.P.; Wade, J.T. ChIP-Seq for Genome-Scale Analysis of Bacterial DNA-Binding Proteins. Methods Mol. Biol. 2015, 1276, 327-340. [CrossRef]

38. Bushnell, B. BBMap Short-Read Aligner, and Other Bioinformatics Tools. 2015. Available online: https://sourceforge.net/ projects/bbmap/ (accessed on 23 January 2019).

39. Zhang, Y.; Liu, T.; Meyer, C.A.; Eeckhoute, J.; Johnson, D.S.; Bernstein, B.E.; Nussbaum, C.; Myers, R.M.; Brown, M.; Li, W.; et al. Model-based Analysis of ChIP-Seq (MACS). Genome Biol. 2008, 9, R137-R139. [CrossRef] [PubMed]

40. Machanick, P.; Bailey, T.L. MEME-ChIP: Motif analysis of large DNA datasets. Bioinformatics 2011, 27, 1696-1697. [CrossRef] [PubMed] 
41. Münch, R.; Hiller, K.; Grote, A.; Scheer, M.; Klein, J.; Schobert, M.; Jahn, D. Virtual Footprint and PRODORIC: An integrative framework for regulon prediction in prokaryotes. Bioinformatics 2005, 21, 4187-4189. [CrossRef]

42. Kopylova, E.; Noé, L.; Touzet, H. SortMeRNA: Fast and accurate filtering of ribosomal RNAs in metatranscriptomic data. Bioinformatics 2012, 28, 3211-3217. [CrossRef] [PubMed]

43. Anders, S.; Pyl, P.T.; Huber, W. HTSeq-A Python framework to work with high-throughput sequencing data. Bioinformatics 2014, 31, 166-169. [CrossRef] [PubMed]

44. Love, M.I.; Huber, W.; Anders, S. Moderated estimation of fold change and dispersion for RNA-seq data with DESeq2. Genome Biol. 2014, 15, 550. [CrossRef]

45. McElroy, K.E.; Hui, J.G.K.; Woo, J.K.K.; Luk, A.W.S.; Webb, J.; Kjelleberg, S.; Rice, S.A.; Thomas, T. Strain-specific parallel evolution drives short-term diversification during Pseudomonas aeruginosa biofilm formation. Proc. Natl. Acad. Sci. USA 2014, 111, E1419-E1427. [CrossRef] [PubMed]

46. Schrodinger, LLC. The PyMOL Molecular Graphics System, Version 2.4.

47. Massad, T.; Skaar, K.; Nilsson, H.; Damberg, P.; Henriksson-Peltola, P.; Haggård-Ljungquist, E.; Högbom, M.; Stenmark, P. Crystal structure of the P2 C-repressor: A binder of non-palindromic direct DNA repeats. Nucleic Acids Res. 2010, 38, 7778-7790. [CrossRef]

48. Maiti, R.; Van Domselaar, G.H.; Zhang, H.; Wishart, D.S. SuperPose: A simple server for sophisticated structural superposition. Nucleic Acids Res. 2004, 32, W590-W594. [CrossRef]

49. Meisner, J.; Goldberg, J.B. The Escherichia coli rhaSR-PrhaBAD Inducible Promoter System Allows Tightly Controlled Gene Expression over a Wide Range in Pseudomonas aeruginosa. Appl. Environ. Microbiol. 2016, 82, 6715-6727. [CrossRef] [PubMed]

50. Alonso, B.; Fernández-Barat, L.; Di Domenico, E.G.; Marín, M.; Cercenado, E.; Merino, I.; De Pablos, M.; Muñoz, P.; Guembe, M. Characterization of the virulence of Pseudomonas aeruginosa strains causing ventilator-associated pneumonia. BMC Infect. Dis. 2020, 20, 1-8. [CrossRef]

51. Berngruber, T.W.; Weissing, F.J.; Gandon, S. Inhibition of Superinfection and the Evolution of Viral Latency. J. Virol. 2010, 84, 10200-10208. [CrossRef]

52. Bednarz, M.; Halliday, J.A.; Herman, C.; Golding, I. Revisiting Bistability in the Lysis/Lysogeny Circuit of Bacteriophage Lambda. PLOS ONE 2014, 9, e100876. [CrossRef]

53. Fernández, L.; Breidenstein, E.B.M.; Song, D.; Hancock, R. Role of Intracellular Proteases in the Antibiotic Resistance, Motility, and Biofilm Formation of Pseudomonas aeruginosa. Antimicrob. Agents Chemother. 2011, 56, 1128-1132. [CrossRef] [PubMed]

54. Oppenheim, A.B.; Kobiler, O.; Stavans, J.; Court, D.L.; Adhya, S. Switches in Bacteriophage Lambda Development. Annu. Rev. Genet. 2005, 39, 409-429. [CrossRef]

55. Ptashne, M. A Genetic Switch, Third Edition, Phage Lambda Revisited; Cold Spring Harbor Laboratory Press: Hamlet in Long Island, NY, USA, 2004.

56. Addy, H.S.; Askora, A.; Kawasaki, T.; Fujie, M.; Yamada, T. The Filamentous Phage фRSS1 Enhances Virulence of Phytopathogenic Ralstonia solanacearum on Tomato. Phytopathology 2012, 102, 244-251. [CrossRef]

57. Cheng, C.-M.; Wang, H.-J.; Bau, H.-J.; Kuo, T.-T. The primary immunity determinant in modulating the lysogenic immunity of the filamentous bacteriophage cf. J. Mol. Biol. 1999, 287, 867-876. [CrossRef] [PubMed]

58. Quinones, M.; Kimsey, H.H.; Waldor, M.K. LexA Cleavage Is Required for CTX Prophage Induction. Mol. Cell 2005, 17, 291-300. [CrossRef]

59. Kimsey, H.H.; Waldor, M.K. The CTX $\phi$ Repressor RstR Binds DNA Cooperatively to Form Tetrameric Repressor-Operator Complexes. J. Biol. Chem. 2004, 279, 2640-2647. [CrossRef]

60. Hay, I.D.; Lithgow, T. Filamentous phages: Masters of a microbial sharing economy. EMBO Rep. 2019, 20, e47427. [CrossRef] [PubMed] 